\title{
A Design Methodology and Various Performance and Fabrication Metrics Evaluation of 3D Network-on-Chip with Multiplexed Through-Silicon Vias
}

\author{
Mostafa Said ${ }^{1}$, Ahmed Shalaby ${ }^{2}$, Farhad Mehdipour ${ }^{3}$, Morteza Biglari-Abhari ${ }^{4}$, Mohamed El-Sayed ${ }^{2}$ \\ ${ }^{1}$ Department of Electrical Engineering, Faculty of Engineering, Assiut University, Assiut, Egypt \\ ${ }^{2}$ Department of Electronics and Communications, Egypt-Japan University of Science and Technology (E-JUST), Alexandria, Egypt \\ ${ }^{3}$ E-JUST Center, Graduate School of Information Science and Electrical Engineering, Kyushu University, Fukuoka, Japan \\ ${ }^{4}$ Department of Electrical \& Computer Engineering, University of Auckland, Auckland, New Zealand \\ Email: mostafa.saied@ejust.edu.eg, Ahamed.Shalaby@ejust.edu.eg, farhad@ejust.kyushu-u.ac.jp, \\ m.abhari@auckland.ac.nz,m.ragab@ejust.edu.eg
}

\begin{abstract}
The use of short Through-Silicon Vias (TSVs) in 3D integration Technology introduces a significant reduction in routing area, power consumption, and delay. Although, there are still several challenges in 3D integration technology; mainly low yield, which is a direct result of extra fabrication steps of TSVs. Therefore, reducing TSV count has a considerable effect on improving yield and hence reducing cost. A TSV multiplexing technique called TSVBOX was introduced in [1] to reduce the TSV count without affecting the direct benefits of TSVs. Although, the TSVBOX introduces some delay to the signals to be multiplexed, this delay effect of TSV multiplexing is not addressed yet. In this paper, we analyze the TSVBOX timing requirements and propose a design methodology for TSVBOXbased 3D Network-on-Chip (NoC). Then performance and power comparisons are conducted to investigate the direct effects of TSV multiplexing on these two metrics. After that the basic fabrication metrics are compared to investigate the effect of the proposed design methodology on yield and cost. We show that the TSVBOX extremely enhances the fabrication metrics at minimal degradation in performance and power consumption, especially for Hotspot-like traffic patterns.
\end{abstract}

\section{INTRODUCTION}

Conventional 2D integration proves to have many limitations for nowadays large systems needs. For example, long wires increase power consumption and routing area. Also, it adds great difficulty to distribute the clock signal with minimum delay for large systems. Such problems make 2D integration unable to follow Moore's law any more [2].

On the other side, 3D integration is an emerging technology that can mitigate the main limitations of conventional 2D integration. However there are still some challenges that need more and more focus and research work to make such promising technology mature and reliable. One of these challenges is the reliability issues in terms of yield and cost. 3D-ICs show very low yield due to extra fabrication steps for bonding dies or wafers to each other to create the 3D stack. These extra steps may result in faulty TSVs due to misalignment of TSVs, partially filled ones, etc. [3]. What makes the situation worse, is that the probability of having faulty TSVs increases as the total number of TSVs increases. Hence, finding a technique that reduces TSV count without affecting the benefits gained by $3 \mathrm{D}$ integration is very important. In [1, 4, 5] the TSV count has been reduced by multiplexing, serialization, or virtualization, respectively. The TSV multiplexing technique introduced in [1] reduces the number of TSVs by half, by multiplexing each two 3D signal ${ }^{1}$ into one signal and passing this signal through one TSV instead of two as in the conventional 3DICs. Therefore almost 50\% reduction in the number of TSVs is achieved. Due to the significant reduction in TSV count, the analysis done in [1] on yield has revealed very high improvement over conventional 3D-ICs.

The investigation in [1] covered area, yield, cost, and power consumption analysis. In [6, 7], other physical effects of reducing TSV count are studied. In [6], the impact of reducing TSV count on maximum temperature is investigated, and as expected the maximum temperature increases as the TSV count decreases. TSVs are usually fabricated using low thermal resistivity materials such as copper or tungsten [2]. Therefore reducing TSV count will increase the total thermal resistance of the 3D stack. In [7] the residual thermal-stress impact created during bonding process in fabrication has been studied carefully to accurately determine the Keep-Out-Zone area overhead around TSVs in order to accurately estimate the yield of the 3D stack.

The TSVBOX uses extra selection signal $(S)$ to control the multiplexer (MUX) and the demultiplexer (DeMUX). This $S$ signal introduces some delay to one of the multiplexed signals besides the parasitics of the TSVBOX itself. Such delay may affect the functional validity of the system to be implemented using TSVBOX. Although, [1, 6, 7] address most of the issues related to TSVs multiplexing and show the advantages and limitations of this technique, the timing requirements and the design methodology based on TSV multiplexing have not

\footnotetext{
${ }^{1} \mathrm{~A} 3 \mathrm{D}$ signal is the signal that traverses from one layer to another in the 3D stack
} 
been studied yet. None of the above related works shows any system implementation of a circuit using TSVBOX, so its functionality and applicability in system level have not been proven yet.

Due to its scalability and novelty as a multicore communication architecture for future multiprocessor SoCs, 3D NoC is selected as our target system architecture for applying the TSVBOX technique. In this paper, timing requirements for TSVBOX-based 3D NoC are investigated so that the delay introduced by the TSVBOX is mitigated and the performance degradation and incorrect operation are avoided. Also, the design methodology for the TSVBOX-based 3D NoCs is introduced. For the sake of comparison, two versions of 3D NoCs are introduced, one based on conventional 3D integration without TSV multiplexing, while the other version is TSVBOX-based. Finally, the main aspects of the NoC architecture in terms of performance and power are investigated under different simulation scenarios.

The contribution of this paper can be summarized in the following points:

- A Low-level circuit model for TSVBOX is introduced, in order to estimate the TSVBOX delay and power consumption; showing possible RC parasitics of its different components.

- Studying the timing requirements of the TSVBOX, determining the selection signal properties and its relation to the main clock signal in the case of 3D NoCs.

- Introducing a complete design methodology with detailed steps required to design a 3D NoC involving TSVBOX.

- Investigating the most important aspects of the 3D NoC; performance and power consumption, showing the cases at which the TSVBOX does not affect these metrics.

- Proposing analytical models to compare the basic fabrication metrics; yield and cost of the conventional and TSVBOX-based 3D NoCs.

Finally, this work is an extensive extension of the work of [8] in many ways. All differences between this work and the work of [8] is included in Table I According to our estimation the extra work is more than $60 \%$ of the work of [8].

The rest of this paper is organized as follows: Section II presents the details of the TSVBOX technique. Section III explores the architecture and design of the target 3D NoC and the 3D router used. Sections IV and Vhighlight the TSVBOX parasitic model and various design aspects. Section VI introduces the TSVBOX timing requirements analysis and the TSVBOX-based 3D NoC design methodology. The scalability analysis is investigated in Section VII while the fabrication yield and cost are analyzed in Section VIII Simulation results are discussed and presented in Section IX Finally, Section $\mathrm{X}$ concludes the paper.

\section{TSVBOX}

Fig. 1a shows the TSVBOX structure. As shown in Fig. 1b. the two inputs of the TSVBOX multiplexer (MUX) are the two signals $\left(V_{1}, V_{2}\right)$ that are supposed to be multiplexed through single TSV. The $S$ signal (Fig. 1c) is the signal that controls MUX and DeMUX, and its clocking period $T_{S}$ is at least equivalent to double the delay of the TSVBOX $\left(T_{d-T S V B O X}\right)$, where $T_{d-T S V B O X}$ is the delay from the input point $V_{1}\left(V_{2}\right)$ to the output point $V_{1}^{\prime}\left(V_{2}^{\prime}\right)$. Assuming that during the first half cycle $V_{2}$ is selected, the TSVBOX circuit will hold the charge of $V_{2}$ during the second half cycle, therefore

$$
V_{2}^{\prime}(t)=V_{2}\left(t-\frac{T_{S}}{2}\right)
$$

During the second half cycle similar behavior for $V_{1}{ }^{\prime}$ is repeated but with another time shift equal to $\frac{T_{S}}{2}$ due to the waiting of the selection process of the first half cycle, so at the end we have

$$
V_{1}^{\prime}(t)=V_{1}\left(t-T_{S}\right)
$$

Fig. 2 shows all the TSVBOX signals and how $V_{1}$ and $V_{2}$ are affected with the TSVBOX delays. For more details about the TSVBOX functionality refer to [1]. In [1] it is assumed that the delay incurred by the TSVBOX $T_{S} \ll T_{C L K}$, therefore this delay can be neglected and there would be no incorrectness issues in reading the multiplexed voltages. In real application this situation is feasible for example when both 3D paths of $V_{1}{ }^{\prime}$ and $V_{2}{ }^{\prime}$ are not part of the critical path, and their delay is at least less than the critical path delay by $T_{S}$. However, the general situation when $T_{S}$ is comparable to the clock signal is not addressed in [1]. Therefore, there are some timing requirements that the TSVBOX must fulfill for the sake of correct operation. In this paper all conditions or requirements will be studied for 3D NoC architecture.

\section{The TARget 3D NoC ARChitecture}

Fig. 3 shows the target 3D NoC architecture, which relies on a $4 \times 4 \times 4$ mesh topology. In [9] an 80 core $\mathrm{NoC}$ was presented, so our target NoC matches the trend of NoC domain. For simplicity, each router has core concentration of one [10], which means only one processor core is connected to the local port of the router. To achieve strong fairness between internal requests of the router, separable allocation is adopted with Round-Robin arbitration [11]-[12]. The size of each injected packet is five flits, and each flit in turn is 64 bits. The head flit contains the routing information while the others are supposed to carry the data. We choose wormhole switching and XYZ deterministic routing to be the $3 \mathrm{D}$ NoC switching technique and routing algorithm [13], respectively. Each input port in the router contains two virtual channels of size one flit while the local virtual channel buffers are assumed to be with infinite size to serve isolating traffic injection process from the NoC. Therefore the delay after generating the flit and before entering source buffer is accounted for as well as the delay in the source queue buffers [14]. According to [15, 16] the total system area can be assumed $400 \mathrm{~mm}^{2}$, thus the area of each layer of the target 3D NoC can be assumed $10 \times 10 \mathrm{~mm}^{2}$. Hence the length of the horizontal interconnect wires between two neighbor routers are $2.5 \mathrm{~mm}$ [16]. For the vertical interconnects, we choose the TSV capacitance to be a parameter in our simulations. The change in TSV capacitance reflects the change in TSV length 
TABLE I: Differences and extra contributions of this extended work in comparison to the work of [8].

\begin{tabular}{|c|c|c|}
\hline Facets of comparison & Work of [8] & This paper \\
\hline 3D NoC adopted & $3 \times 3 \times 2$ & $4 \times 4 \times 4$ \\
\hline Switiching technique & Store-And-Forward & Wormhole \\
\hline Virtual channels (VCs) & Single buffer per port & $2 \mathrm{VCs}$ per port \\
\hline Technology adopted & $180 \mathrm{~nm}$ & More finer $65 \mathrm{~nm}$ technology \\
\hline TSV capacitance & Fixed at $15 \mathrm{fF}$ & $\begin{array}{l}\text { Considered as a parameter with } 15 \mathrm{fF} \text { to } 500 \mathrm{fF} \\
\text { to reflect different TSV technologies }\end{array}$ \\
\hline TSVBOX multiplexing ratio used & Fixed at $2 \times 1$ & Considered as a general parameter $N_{M U X} \times 1, N_{M U X} \geq 2$ \\
\hline Yield and cost analysis & - & Included in Section $\overline{\mathrm{VIII}}$ \\
\hline $\begin{array}{l}\text { Analytical analysis on the effect of different } \\
\text { traffic patterns on the performance of TSVBOX }\end{array}$ & - & Included in Subsection VII-D \\
\hline Power consumption evaluation & - & Included in Subsection IX-E \\
\hline Scalability analysis & - & Included in Section VII \\
\hline
\end{tabular}

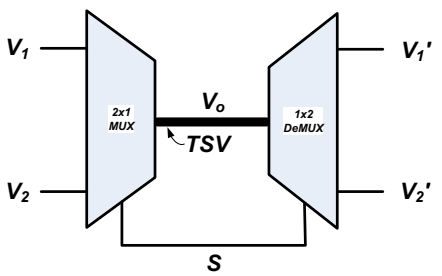

(a) TSVBOX schematic

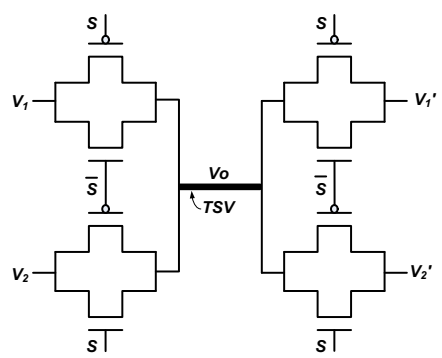

(b) TSVBOX circuit

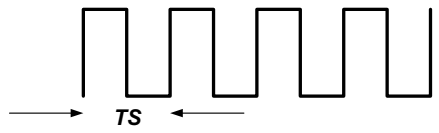

(c) Selection signal $S$

Fig. 1: (a) TSVBOX schematic, (b) TSVBOX circuit implementation, (c) Selection signal $S$.

and the resistivity of the substrate bulk used which in turn reflects different 3D integration technologies [17]-[20].

The data bus width $N_{B W}$ is assumed to be equivalent to the flit size, as shown in Figs. 5a $5 \mathrm{~b}$. For the conventional 3D NoC, the whole 3D data bus width is $N_{B W}+2$, where the extra two bits are required for the handshaking communication protocol which needs request $(R E Q)$ and acknowledgement $(A C K)$ signals [21]. For the TSVBOX case, the data bits of the packet are multiplexed and hence $\frac{N_{B W}}{2}+2$ TSVs are required, including $R E Q$ and $A C K$ signals. However for each vertical bus width two extra TSVs are required to transfer the $S$ signal and its inverted version $\bar{S}$. Therefore, the vertical connection bus width is $\frac{N_{B W}}{2}+4$ for the TSVBOXbased 3D NoC. Any two neighbor routers are connected by

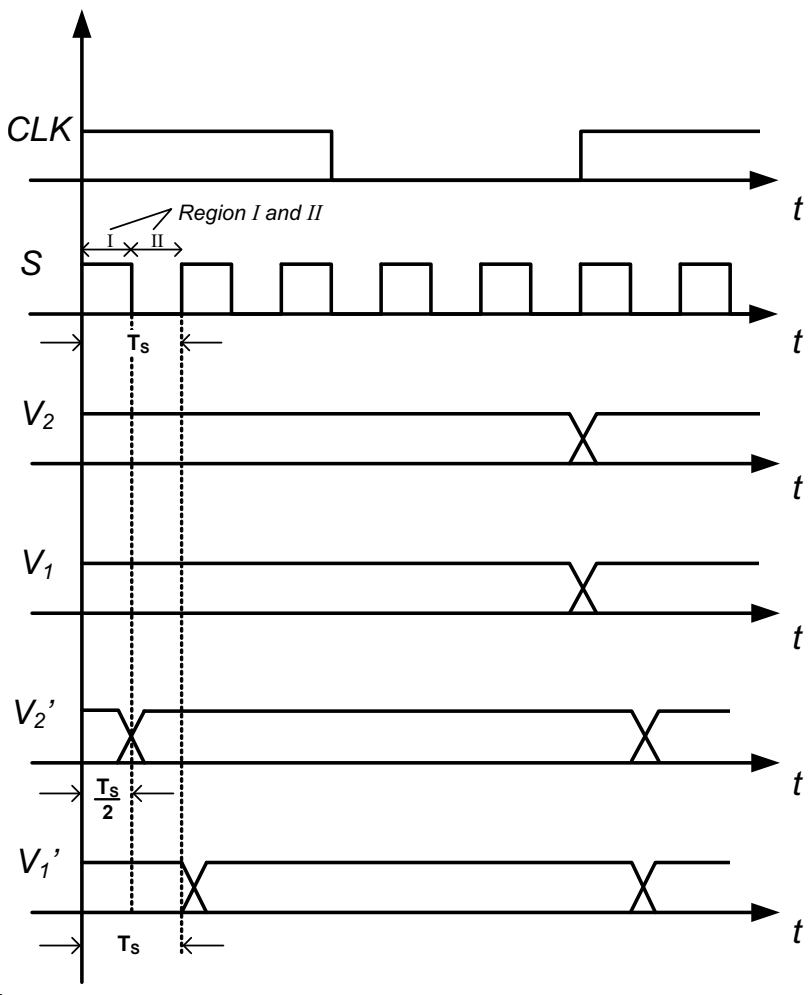

Fig. 2: Various TSVBOX signals; $S(t), V_{1}(t), V_{2}(t)$, $V_{1}^{\prime}(t)$, and $V_{2}^{\prime}(t)$.

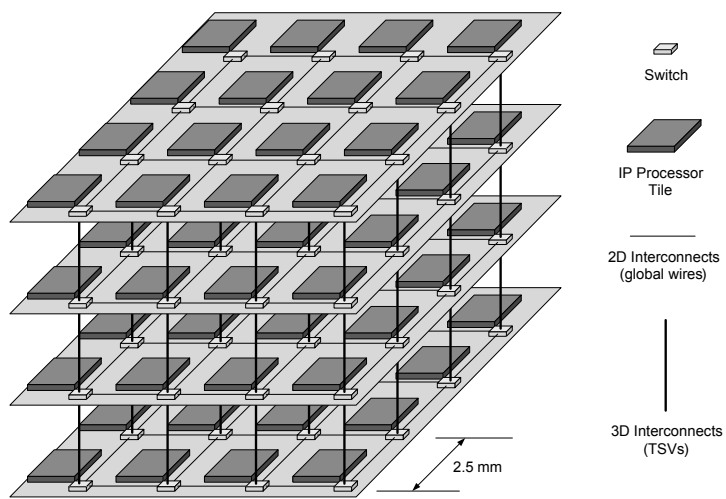

Fig. 3: $4 \times 4 \times 43 \mathrm{D}$ NoC architecture. 
two opposite unidirectional channels. Thus the vertical port contains $2\left(N_{B W}+2\right)$ TSVs for conventional 3D NoC, and $2\left(\frac{N_{B W}}{2}+4\right)$ for TSVBOX case.

Since SPICE models for such system would be too complicated and time consuming either in design or simulation, SystemC-A is used for our 3D NoC implementation. SystemC supported by the Open SystemC Initiative (OSCI) [22] is an open source language available to meet the ever-increasing needs of system-level design and $\mathrm{SoC}$ technologies. Using SystemC-A, high- and low-levels of implementation can be done together for a system. For processor cores, routers, and intra-layer interconnects, we use behavioral system implementation, while for inter-layer interconnects (the vertical connections), we rely on a low-level circuit implementation to be able to estimate delays and power consumption.

\section{Modeling}

As shown in Fig. 4 4 , the 3D signal is assumed to pass through an input inverter driver, a global wiring segment in the first layer, a TSV, a global wiring segment in the second layer, and an output inverter driver. The output inverter driver is assumed always $1 \mathrm{x}$-inverter (minimum size inverter). For the TSV and wiring circuit models, the models introduced in [2, 23] are used, which are shown in Figs. 4(b,c), respectively.

The TSVBOX is composed of MUX and DeMUX circuits and a TSV in between. The MUX or the DeMUX is composed of two transmission gates and each transmission gate is composed of two transistors. Therefore to model TSVBOX, a transistor circuit model that depicts different transistors' $\mathrm{RC}$ parasitics is required. Referring to [24], the transistor parasitics can be modeled as shown in Fig. 4(d,e). The parasitics of this model are: $O N$ resistance of $N M O S\left(R_{o n N}\right)$, $O N$ resistance of PMOS $\left(R_{o n P}\right), N M O S$ source/drain-bulk capacitance $\left(C_{s b N} / C_{d b N}\right), P M O S$ source/drain-bulk capacitance $\left(C_{s b P} / C_{d b P}\right), N M O S$ gate capacitance $\left(C_{g N}\right)$, and $P M O S$ gate capacitance $\left(C_{g P}\right)$. According to [24], $C_{s b N}=C_{d b N}=C_{N}$ for $N M O S$ and $C_{s b P}=C_{d b P}=C_{P}$ for PMOS. Also, for equivalent

TABLE II: Transistor and other miscellaneous parasitics for $65 \mathrm{~nm}$.

\begin{tabular}{|l|c|c|}
\hline $\begin{array}{c}\text { Transistor } \\
\text { parasitic }\end{array}$ & Unit & $65 \mathrm{~nm}$ \\
\hline$C_{g P}$ & $\mathrm{fF}$ & 0.0689 \\
\hline$C_{g N}$ & $\mathrm{fF}$ & 0.0689 \\
\hline$C_{s b P}$ & $\mathrm{fF}$ & 0.0832 \\
\hline$C_{s b N}$ & $\mathrm{fF}$ & 0.0819 \\
\hline$C_{d b P}$ & $\mathrm{fF}$ & 0.0832 \\
\hline$C_{d b N}$ & $\mathrm{fF}$ & 0.0819 \\
\hline$R_{o n P}$ & $\mathrm{k} \Omega$ & 44.462 \\
\hline$R_{o n N}$ & $\mathrm{k} \Omega$ & 21.077 \\
\hline$C_{W}$ & $\mathrm{fF}$ & 25 \\
\hline$R_{W}$ & $\mathrm{k} \Omega$ & 0.04132 \\
\hline $\mid V_{t h P}$ & $\mathrm{~V}$ & 0.39 \\
\hline$V_{t h N}$ & $\mathrm{~V}$ & 0.4 \\
\hline$V_{D D}$ & $\mathrm{~V}$ & 1 \\
\hline$C_{L}$ & $\mathrm{fF}$ & 1.3 \\
\hline$C_{p}$ & $\mathrm{fF}$ & 1.5 \\
\hline
\end{tabular}

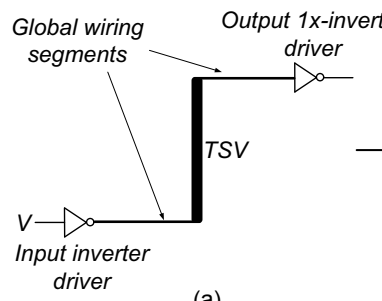

(a)

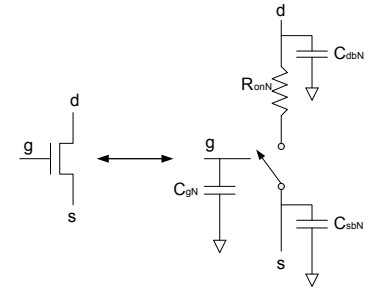

(d)

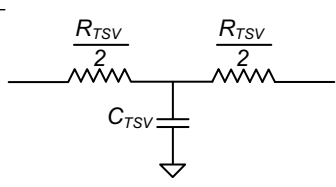

(b)

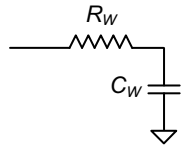

(c)
Fig. 4: (a) The 3D signal path and circuit models of (b) TSV, (c) global wiring, (d) NMOS, and (e) PMOS.

NMOS and PMOS sizes, both $N M O S$ and $P M O S$ gate capacitances' values are equivalent so $C_{g P}=C_{g N}=C_{g}$.

\section{A. Conventional 3D-IC 3D signal path modeling}

The conventional 3D-IC 3D signal path is shown in Fig. 6 where the signal is assumed to pass through an inverter driver (represented by its $O N$ resistance $R_{d r-C o n v}$ ), a global wiring segment in the first layer, a TSV, a global wiring segment in the second layer, and a load capacitance which is assumed to be the input gate capacitance of a $1 \mathrm{x}$-inverter driver in the second layer.

\section{B. TSVBOX $3 D$ signal path modeling}

Fig. 7 shows the TSVBOX circuit model. It is similar to the circuit of the conventional 3D signal path, the difference is that the equivalent RC parasitic circuit of the transistors in MUX and DeMUX are involved. Since there is no transistor models in SystemC-A, the transistors of the transmission gate are modeled using perfect switches. The $S$ signal controls the upper transmission gates of the MUX and the DeMUX, and its inverted version $\bar{S}$ controls the lower ones. Therefore both the lower and upper transmission gates will switch $O N$ or $O F F$ exclusively as required in the original TSVBOX design. The $S$ signal path shown in Fig. 77, is similar to the conventional 3D signal path. However, since the $S$ signal is driving the gates of the transmission gates, therefore for each $S$ path, there are four gate capacitances $4 C_{g}$ involved in the load; $2 C_{g}$ from MUX and $2 C_{g}$ from DeMUX.

\section{Design Parameters and Parasitics}

In this section, parasitics values and technology parameters are introduced and the design considerations are detailed.

\section{A. Technology parasitics and parameters}

In this study $65 \mathrm{~nm}$ is selected to be our target technology. Technology parasitics and parameters spans the NMOSs' and PMOSs' parasitics shown in Fig. 4 and also their threshold voltages. It also covers the global wiring parasitics which are used for IPs and multicore 3D-ICs [25] and the input and 


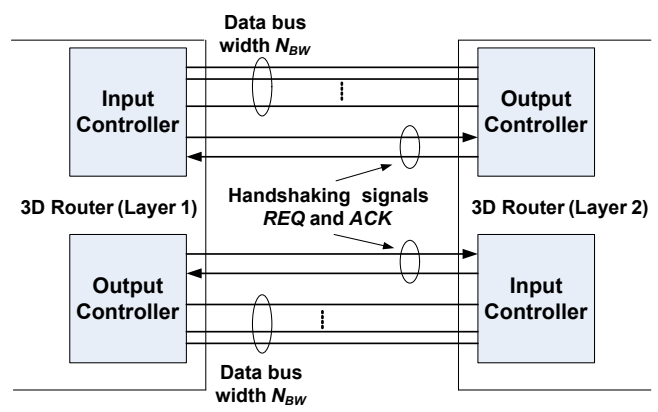

(a)

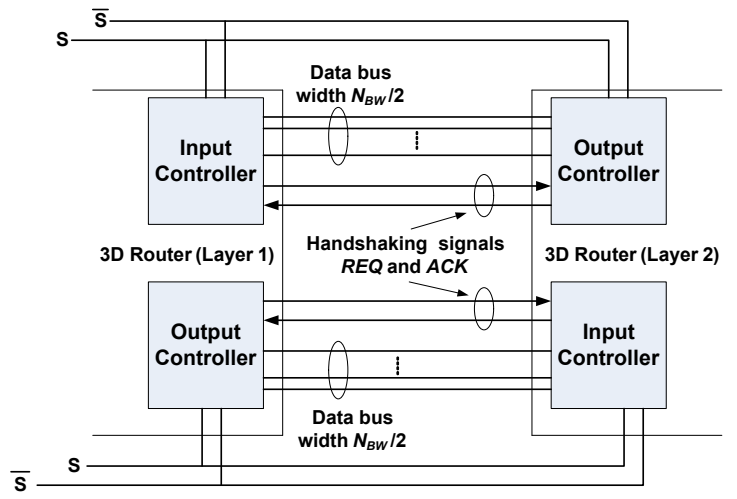

(b)

Fig. 5: Full duplex transmission for (a) conventional and (b) TSVBOX-based 3D NoC.

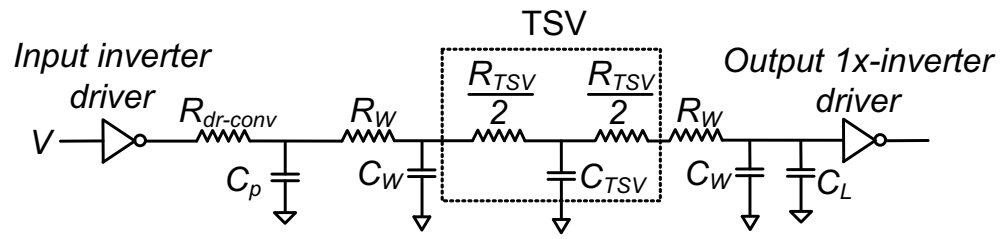

Fig. 6: Conventional 3D NoC 3D signal path.

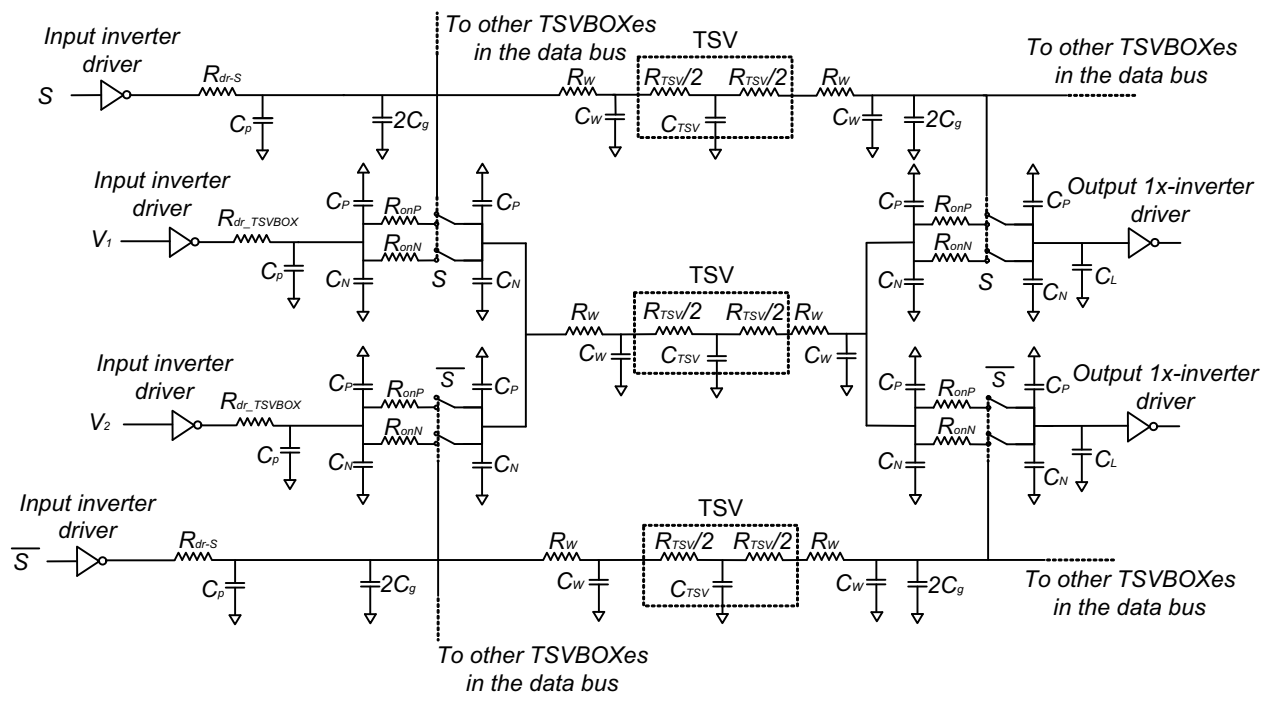

Fig. 7: TSVBOX-based 3D NoC 3D signal path.

the output capacitances of 1x-inverter driver shown in Fig. 4 4 . Table II] shows all the values used in this study [24-26], noting that the length of the wires is assumed $200 \mu \mathrm{m}$ similar to [17].

For the TSV technology, the experiments run once for $C_{T S V}=15 \mathrm{fF}$ and another for $C_{T S V}=500 \mathrm{fF}$ to cover the whole range of TSV capacitances and technologies, while the maximum value for $R_{T S V}=1 \Omega$ is selected for all cases [17].

\section{B. Transmission gate transistors}

According to [27], transmission gate transistors are usually selected to have minimum size. Also, as stated in [27], there is no need to decrease $R_{o n P}$, hence $W_{P}=L_{P}$ and $K_{N}=K_{P}=1$ (the sizes of NMOS and PMOS transistors, respectively) are our design choices for the transmission gate transistors.

\section{Threshold voltage selection for the drivers}

There are two input thresholds: $V_{i n L-\max }$ and $V_{i n H-\min }$. $V_{\text {inL-max }}$ is the maximum low input voltage required to switch PMOS ON and NMOS OFF at the same time. Therefore if $V_{i n} \leq V_{i n L-\max }, N M O S$ will be $O F F$ and PMOS will be $O N$. The other threshold voltage is $V_{i n H-m i n}$, which is the minimum high input voltage required to switch 
NMOS ON and PMOS OFF at the same time, therefore if $V_{\text {in }} \geq V_{\text {inH-min }}, N M O S$ will be $O N$ and $P M O S$ will be $O F F$. Depending on the previous definitions, the thresholds can be selected as follows

$$
V_{i n L-\max }=V_{t h N}, V_{i n H-\min }=V_{D D}-\left|V_{t h P}\right|
$$

\section{TIMING REQUIREMENTS' ANALYSIS}

In this section all timing analysis related to TSVBOX-based 3D NoC is discussed. Timing requirements' analysis include 3D path delays, how to choose the selection signal period $T_{S}$, and the relation between $S$ and the main clock signal.

\section{A. 3D signal path Elmore-delay model}

The 3D signal path delay for data and $S$ signals in conventional and TSVBOX-based 3D NoCs can be approximated using first order Elmore-delay model [24]. The delay for data signals is the time required for the 3D data signal to reach $V_{i n H-\min }=V_{D D}-\left|V_{t h P}\right|$. The 3D data signals passing through the conventional or the TSVBOX 3D paths are to be destined to a $1 \mathrm{x}$-inverter driver load, as shown in Figs. (67), respectively. For $S$ signal, the delay is the time required for $S$ to reach $\max \left(V_{t h N},\left|V_{t h P}\right|\right)$, because $S$ is destined to the transmission gate transistors (PMOS or NMOS). Hence the voltage required for $S$ signal to switch either $N M O S$ or $P M O S$ $O N$ is $V_{t h N}$ or $\left|V_{t h P}\right|$, respectively. The maximum of these two voltages is selected to meet both conditions.

According to Section $\mathrm{V}$, the wiring and TSV resistances are in order of ohms, therefore their contribution to the total delay is negligible. Thus these resistances will be ignored to simplify the delay analysis in this paper.

1) Conventional $3 \mathrm{DNoC} 3 \mathrm{D}$ signal delay: Referring to Fig. 6, the conventional 3D NoC 3D signal path delay $\left(T_{d-C o n v}\right)$ can be approximated using Elmore-delay as follows:

$T_{d-C o n v}=\ln \frac{V_{D D}}{\left|V_{t h P}\right|} \cdot\left(R_{d r-C o n v} \cdot\left(C_{p}+2 C_{W}+C_{T S V}+C_{L}\right)\right)$

2) TSVBOX-based $3 D$ NoC $3 D$ signal delay: The TSVBOX has two different paths according to the values of the $S$ signal. Fig. 8 shows the situation when $S=1(\bar{S}=0)$ that $V_{1}$ passes through the TSVBOX, while Fig. 9 shows the other state when $S=0(\bar{S}=1)$ that $V_{2}$ passes. As noticed, both paths are similar, therefore the TSVBOX 3D path delay $\left(T_{d-T S V B O X}\right)$ for both $V_{1}$ and $V_{2}$ is the same. The Elmore-delay for both $V_{1}$ and $V_{2}$ can be approximated according to the following equation

$$
\begin{array}{r}
T_{d-T S V B O X}=\ln \frac{V_{D D}}{\left|V_{t h P}\right|} \cdot\left(R_{d r-T S V B O X} \cdot\left(C_{p}+C_{P N}\right)\right. \\
+\left(R_{d r-T S V B O X}+R_{P N}\right) \cdot\left(4 C_{P N}+2 C_{W}+C_{T S V}\right) \\
\left.+\left(R_{d r-T S V B O X}+2 R_{P N}\right) \cdot\left(C_{P N}+C_{L}\right)\right)
\end{array}
$$

$$
\text { where } C_{P N}=C_{d b P}+C_{d b N}, R_{P N}=\frac{R_{o n P} \cdot R_{o n N}}{R_{o n P}+R_{o n N}}
$$

3) Selection signal delay: The $S$ signal path is shown previously as a part of the TSVBOX in Fig. 7. As shown, each TSVBOX contributes to the load of $S$ signal by $4\left(C_{g N}+C_{g P}\right)$. Then for $\frac{N_{B W}}{2}$ data bus width, the TSVBOXes will contribute with $N_{B W} \cdot\left(C_{g N}+C_{g P}\right)$ in total to the $S$ signal load. As stated in Section $\mathrm{V}$, the transmission gates transistors are equally sized $\left(C_{g N}=C_{g P}=C_{g}\right)$, therefore the total gate load for $S$ signal is $2 N \cdot C_{g}$. The Elmore-delay for $S$ signal to reach $\max \left(V_{t h N},\left|V_{t h P}\right|\right)$ is

$$
\begin{array}{r}
T_{d-S}=\ln \frac{V_{D D}}{V_{D D}-\max \left(V_{t h N},\left|V_{t h P}\right|\right)} \\
\cdot\left(R_{d r-S} \cdot\left(C_{p}+2 C_{W}+C_{T S V}+2 N_{B W} \cdot C_{g}\right)\right)
\end{array}
$$

\section{B. Avoiding concurrent ON state of the TSVBOX switches}

As shown in Fig. 10 . At $t=\frac{T_{S}}{2}, S$ and $\bar{S}$ will start discharging and charging, respectively. However, $\bar{S}$ will reach the $O N$ threshold voltage $\max \left(V_{t h N},\left|V_{t h P}\right|\right)$ at $t=T_{1}$, while $S$ will reach the $O F F$ threshold voltage $\min \left(\mathrm{V}_{t h N},\left|V_{t h P}\right|\right)$ at $t=T_{2}$. This is because $\bar{S}$ is ' 0 ' and $S$ is $V_{D D}$, and ' 0 ' is much closer to $\max \left(V_{t h N},\left|V_{t h P}\right|\right)$, than $V_{D D}$ to $\min \left(\mathrm{V}_{t h N},\left|V_{t h P}\right|\right)$. It can be seen that during $T_{1} \leq t \leq T_{2}$, all the transmission gate switches of the TSVBOX are $O N$ which violates the theoretical behavior of the TSVBOX.

Fig. 11 displays how the above problem could occur as a result of the previously discussed behavior ${ }^{2}$. In this example, $V_{1}$ and $V_{2}$ are supposed to be logic ' 1 ' and ' 0 ', respectively. Although, during $\left[0, \frac{T_{S}}{2}\right] V_{1}^{\prime}$ reaches $\approx 1.32 \mathrm{~V}$ and $V_{2}^{\prime}$ is ' 0 ' and both are acceptable voltage levels $\left(V_{1}^{\prime}>V_{i n H-m i n}\right.$ and $\left.V_{2}{ }^{\prime}<V_{i n L-\max }\right) . V_{1}{ }^{\prime}$ reduces in the period of concurrent operation ( $T_{\text {overlap }}$ ) to $\approx 1.12 \mathrm{~V}$ which is $<V_{\text {inH-min }}$. At $t=T_{2}, S$ reaches the $O F F$ threshold and the switches of $V_{1}$ path become open so $V_{1}^{\prime}$ will continue at $\approx 1.12 \mathrm{~V}$ till $t=T_{S}$ (the instant of reading data of downstream router) therefore it can not be considered as the logic high as expected.

To avoid such problem we must be sure that $V_{1}^{\prime}$ and $V_{2}^{\prime}$ have acceptable voltage levels at $t=T_{S}$; the time of reading the data by the downstream router. A simple solution is to let both $\mathrm{S}$ and $\bar{S}$ to charge and discharge faster in order to tighten the overlap period such that $V_{1}^{\prime}$ and $V_{2}^{\prime}$ discharge to acceptable levels. For instance, in the above illustrated example in Fig. 11 $V_{1}^{\prime}$ and $V_{2}^{\prime}$ should discharge to some voltages $>V_{i n H-m i n}$ and $<V_{\text {inL-max }}$, respectively.

In summary we will calculate $T_{\text {overlap }}$ using two different ways, once by calculating $T_{1}$ and $T_{2}$ and then subtracting $T_{1}$ from $T_{2}$ and in this way $T_{\text {overlap }}$ would be a function of $S$ and $\bar{S}$ drivers' sizes. And the second way is by using the observation that $T_{\text {overlap }}$ should be smaller than or equal the time taken by $V_{1}{ }^{\prime}$ to reach $V_{i n H-\min }$. By equating these two equations together we can determine the driver size of both $S$ and $\bar{S}$ that will result in minimum acceptable $T_{\text {overlap }}$ such that at $t=T_{S} \quad V_{1}^{\prime}$ and $V_{2}^{\prime}$ would have correct and

\footnotetext{
${ }^{2}$ For the sake of better clarity, we derive this example adopting $180 \mathrm{~nm}$ technology, in which $V_{D D}=1.8 \mathrm{~V}, V_{t h N}=0.53 \mathrm{~V}$, and $\left|V_{t h P}\right|=0.51 \mathrm{~V}$. Therefore, voltage levels would be more clear than the case of $65 \mathrm{~nm}$.
} 


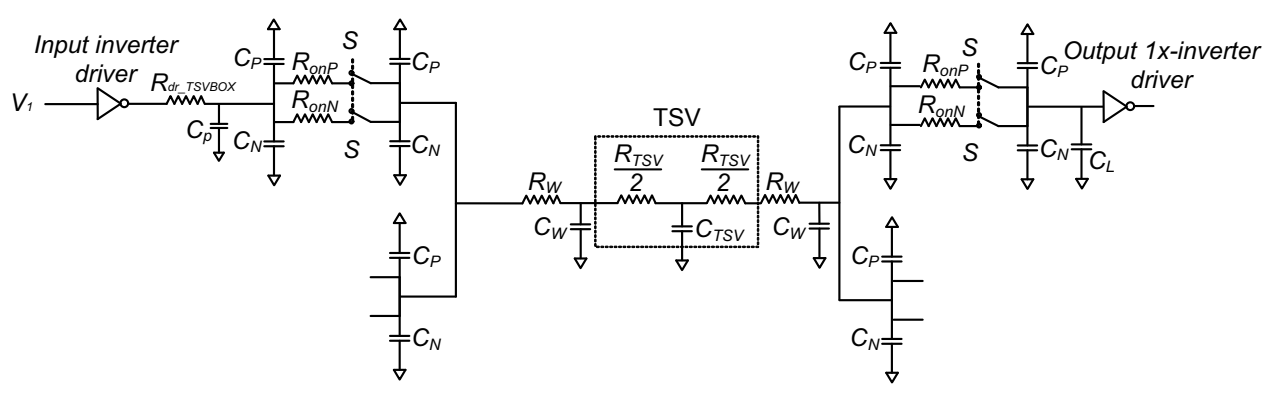

Fig. 8: $V_{1} 3 \mathrm{D}$ path.

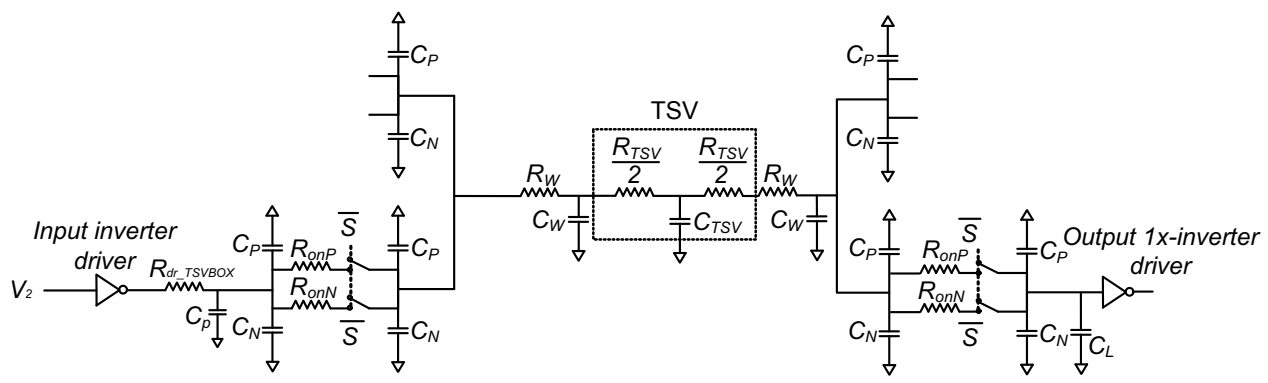

Fig. 9: $V_{2}$ 3D path.

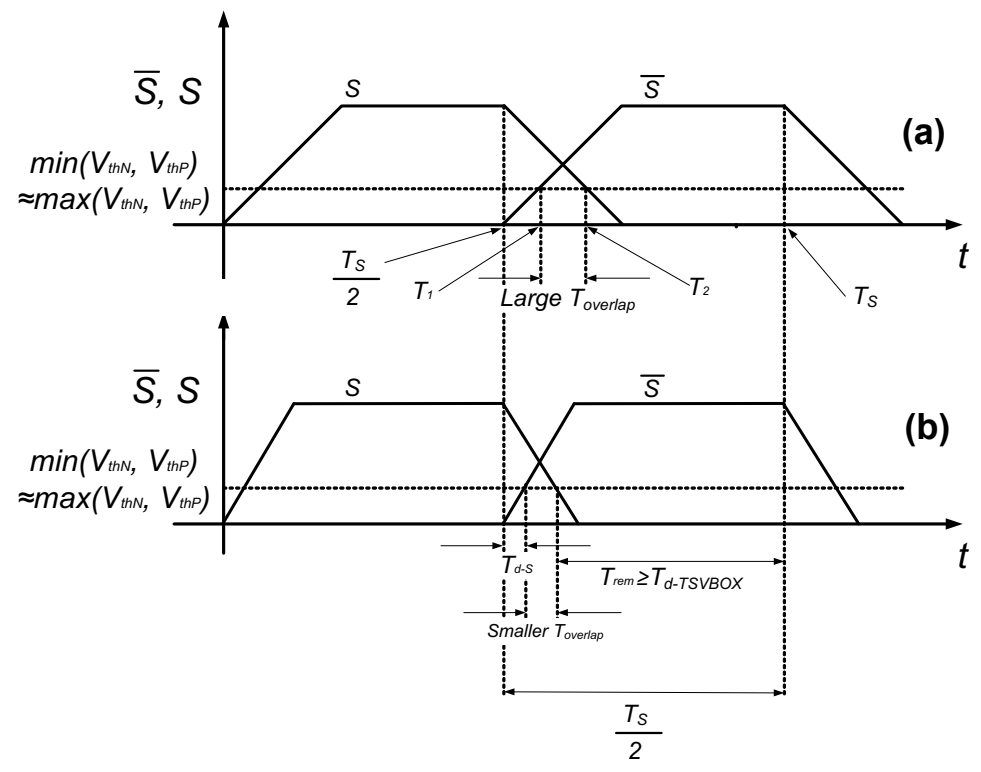

Fig. 10: $S$ and $\bar{S}$ signals before (a) and after (b) increasing driver sizes to avoid negative effects of concurrent $O N$ states of the TSVBOX switches.

acceptable voltage levels. To simplify the following analysis two assumptions are made. First, we choose the same driver size for $S$ and $\bar{S}$, so

$$
R_{d r-\bar{S}}=R_{d r-S}
$$

Second, we will assume that $\frac{T_{S}}{2}$ is very large so that $V_{1}{ }^{\prime}$ charges to $V_{D D}$ not only up to $V_{i n H-\min }$ during $\left[0, \frac{T_{S}}{2}\right]$ and hence the discharging curve would start from $V_{D D}$ not
$V_{\text {inH-min }}$.

Further, for better visualization, Fig. 12 shows a simplified version of the TSVBOX circuit, where $\quad C_{p-P N-1}=C_{p-P N-2}=C_{p}+C_{P N}$, $C_{P N-L-1}=C_{P N-L-2}=C_{P N}+C_{L}$, and the intermediate capacitance $C_{i n t}=4 C_{P N}+2 C_{W}+C_{T S V}$.

Assuming $T_{1}$ is the time taken by $\bar{S}$ to charge to $\max \left(V_{t h N},\left|V_{t h P}\right|\right)$, and $T_{2}$ is the time taken by $S$ to discharge to $\max \left(V_{t h N},\left|V_{t h P}\right|\right)$, therefore

$$
\begin{gathered}
T_{1}=\tau_{\bar{S}} \cdot \ln \frac{V_{D D}}{V_{D D}-\max \left(V_{t h N},\left|V_{t h P}\right|\right)} \\
T_{2}=\tau_{S} \cdot \ln \frac{V_{D D}}{\min \left(V_{t h N},\left|V_{t h P}\right|\right)}
\end{gathered}
$$

And since $S$ and $\bar{S}$ have the same driver sizes, so $\tau_{\bar{S}}=\tau_{S}=R_{d r-S} \cdot\left(C_{p}+2 C_{W}+C_{T S V}+2 N_{B W} \cdot C_{g}\right)$

Then the overlap period can be calculated as

$$
T_{\text {overlap }}=\tau_{S} \cdot \ln \frac{V_{D D}-\max \left(V_{t h N},\left|V_{t h P}\right|\right)}{\min \left(V_{t h N},\left|V_{t h P}\right|\right)}
$$

The second way to calculate $T_{\text {overlap }}$ requires the discharge equation of $V_{1}{ }^{\prime}$ which is not an easy task since during $T_{\text {overlap }}$ $C_{L 1}$ is discharging in all other capacitors in the circuit and at the same time it is charging from the source $V_{1}$ as well as other capacitors. According to our simulation we found that the discharging current from $C_{L 1}$ into $C_{L 2}, C_{p-P N-1}$, $C_{p-P N-2}$, and $C_{i n t}$ is the most dominant, thus we developed the following discharge equation:

$$
V_{1}^{\prime}(t)=V_{f}+\left(V_{i}-V_{f}\right) \cdot \exp \left(\frac{-t}{\tau}\right)
$$




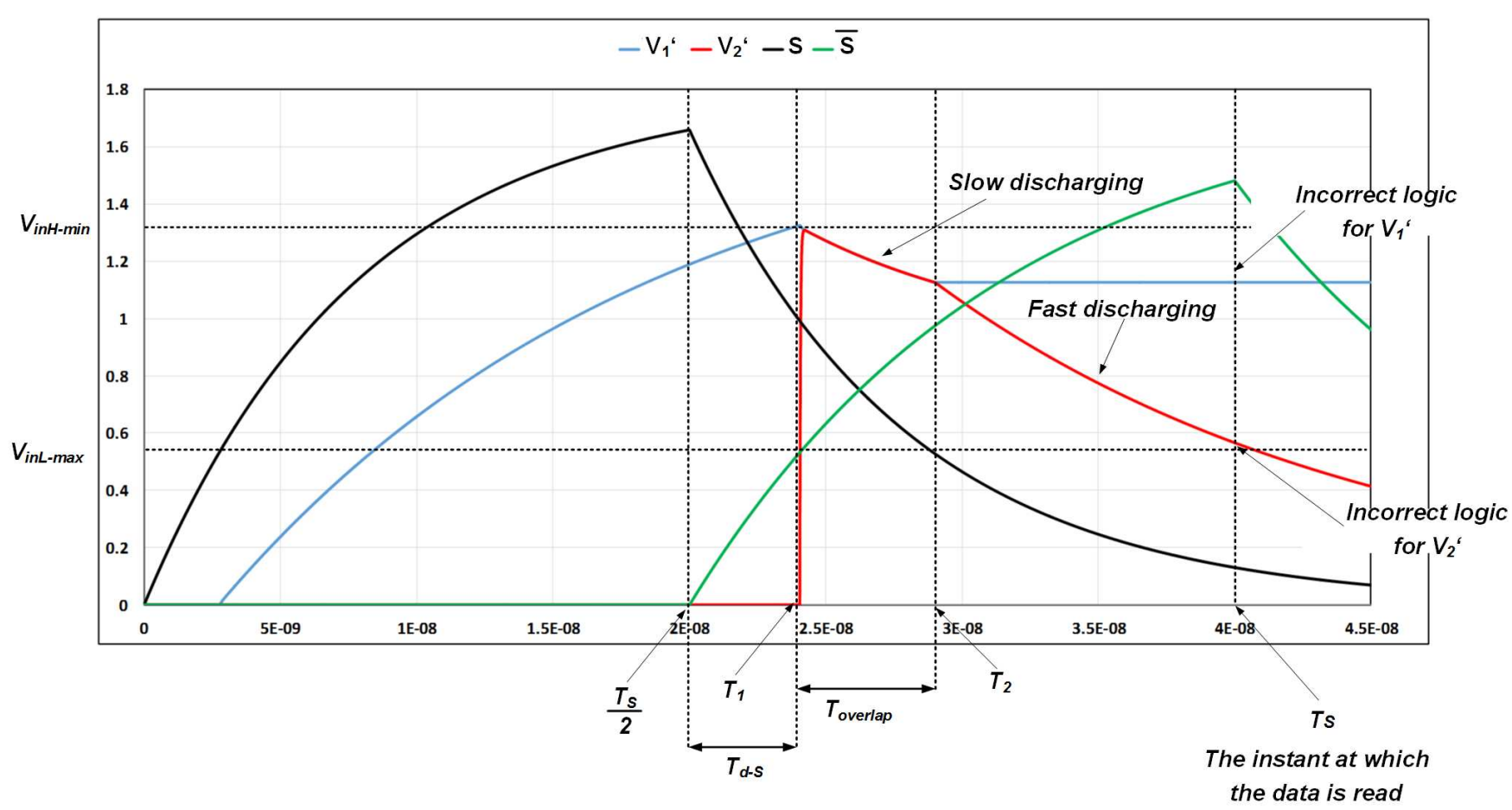

Fig. 11: SystemC-A output signals showing complemented 3D signals multiplexing issues.

where $V_{i}=V_{D D}, V_{f}=\frac{V_{D D}}{2}$, and

$\tau=2 R_{P N} \cdot\left(C_{P N-L-2}+C_{p-P N-1}+C_{p-P N-2}\right)+R_{P N} \cdot C_{i n t}$

To check the accuracy of Eq. 10 we calculate the theoretical value of $V_{1}^{\prime}$ from $\mathrm{Eq} 10$ and compare it with the value gained from simulation using SystemC-A. The error between the theoretical and simulational models does not exceed $8 \%$ which indicates the acceptable accuracy of this discharge equation.

Now using Eq 10 , the time taken by $V_{1}^{\prime}$ to reach $V_{i n H-m i n}$ can be simply determined:

$$
\left.T\right|_{V_{1}{ }^{\prime}=V_{i n H-m i n}}=T_{\text {overlap }}=\tau \cdot \ln \frac{V_{D D}-2 V_{t h p}}{V_{D D}}
$$

Finally, by equating Eqs. 9 and 11 the value of $R_{d r-S}$ and the proper size of the $S$ driver can be determined to avoid the concurrent operation problems.

It is worth mentioning that, the above stated steps assumes an ideal case in which $V_{1}^{\prime}$ reaches $V_{D D}$ during charging while we assume that $V_{i n H-m i n}$ is the enough level to be considered as logic ' 1 ' and $T_{d-T S V B O X}$ is defined according to that. Consequently, the overlap period must be 0 which is realized using zero resistance selection signal driver which means an infinite size inverter driver and this is not possible. To solve such conflict, henceforth we will permit $V_{1}{ }^{\prime}$ and $V_{2}{ }^{\prime}$ to charge to value slightly higher than $V_{i n H-m i n}$. For example in this paper, we choose $V_{1}{ }^{\prime}$ and $V_{2}{ }^{\prime}$ to charge to $1.1 V_{i n H-m i n}$ which is $10 \%$ higher than $V_{i n H-m i n}$. Hence the equations defining

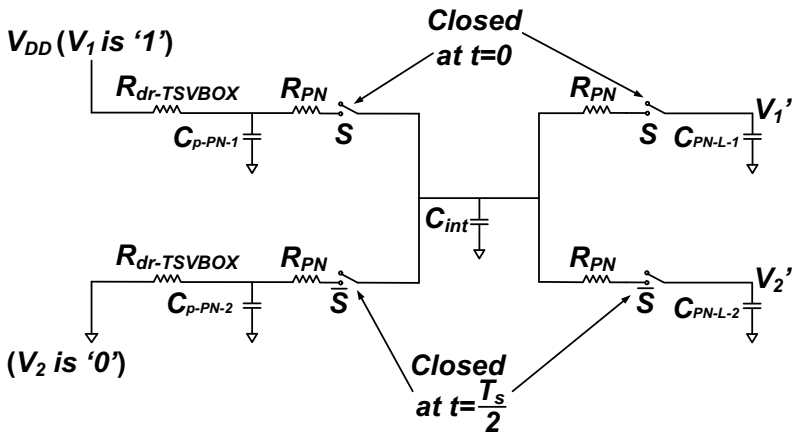

Fig. 12: TSVBOX simplified circuit model.

$T_{d-T S V B O X}$ and $T_{\text {overlap }}$ would be changed to

$$
\begin{array}{r}
T_{d-T S V B O X}=\ln \frac{V_{D D}}{\left|V_{t h P}\right|-0.1\left(V_{D D}-\left|V_{t h P}\right|\right)} \\
\cdot\left(R_{d r-T S V B O X} \cdot\left(C_{p}+C_{P N}\right)+\left(R_{d r-T S V B O X}+R_{P N}\right)\right. \\
\cdot\left(4 C_{P N}+2 C_{W}+C_{T S V}\right)+\left(R_{d r-T S V B O X}+2 R_{P N}\right) \\
\left.\cdot\left(C_{P N}+C_{L}\right)\right)
\end{array}
$$

and

$$
T_{\text {overlap }}=\tau \cdot \ln \frac{V_{D D}-2\left|V_{t h P}\right|+0.2\left(V_{D D}-\left|V_{t h P}\right|\right)}{V_{D D}-2\left|V_{t h P}\right|}
$$

, respectively. Apparently, both delays are higher than the delays of Eqs. 5 and 11 , respectively. 


\section{Minimum duration of the selection signal}

After introducing the driver design of $S(\bar{S})$ in the previous subsection, its clocking period $T_{S}$ can be derived. As shown in Fig. 10, the period of time between $\frac{T_{S}}{2} \leq t \leq T_{S}$, can be divided into three smaller periods:

(1) $T_{d-S}$; the time required by $\bar{S}$ to reach $\max \left(V_{t h N},\left|V_{t h P}\right|\right)$.

(2) $T_{\text {overlap }}$; the permitted overlap period of concurrent $O N$ state of TSVBOX switches.

(3) $T_{r e m}$; the remaining time until $T_{S}$. During this period, $V_{2}^{\prime}$ signal is required to reach an acceptable level ' 0 ' or ' 1 ', therefore we must select $T_{r e m} \geq T_{d-T S V B O X}$.

Based on these observations the minimum clocking period $T_{S-\min }$ for $S(\bar{S})$ control signal can be expressed as follows:

$$
T_{S-\min }=2\left(T_{d-\text { overlap }}+T_{d-S}+T_{d-T S V B O X}\right)
$$

\section{Selection signal generation}

In order to simplify the design procedure, $S$ signal should be derived from the clock signal itself. To simplify more, $T_{S}$ can be selected as an even integer multiple of the clocking period $T_{C L K}$, i.e. $T_{S}=2 n . T_{C L K}$, and $n$ is chosen such that the inequality $T_{S} \geq T_{S-\min }$ is fulfilled. To select $n$, the relation between $T_{S-\min }$ and $T_{C L K}$ must be known first. This inequality has three different cases:

(1) $T_{C L K}<\frac{T_{S-\min }}{2}$ : we can choose $\frac{T_{S}}{2}=n . T_{C L K}, n=2,3$, etc., or in other words $T_{S}=2 n \cdot T_{C L K}{ }^{3}$ and we choose $n$ such that $\frac{T_{S}}{2} \geq \frac{T_{S-\min }}{2}$.

(2) $\frac{T_{S-\min }}{2} \leq T_{C L K}<T_{S-\min }$ : in this case the period of time $T_{C L K}$ is sufficient to pass $V_{1}$ or $V_{2}$ (but not both) through the TSVBOX, therefore $\frac{T_{S}}{2}$ can be chosen to be $T_{C L K}$, or more formally $T_{S}=2 T_{C L K}$ ( $n$ is constant equal 1$)$.

(3) $T_{C L K} \geq T_{S-m i n}$ : in this case the period of time $T_{C L K}$ is sufficient to pass $V_{1}$ then $V_{2}$ (or vice versa), serially. Therefore we can choose $T_{S}=T_{C L K}$ ( $n$ is constant equal to $\frac{1}{2}$ ).

As noticed from the above cases, the TSVBOX degrades the performance of the 3D NoC in cases (1) and (2), where we need more than one clock cycle to transfer the data per vertical hop. In contrast, in case (3) the TSVBOX shows the same performance as conventional 3D NoC, because the multiplexing operation needs only a clock cycle to transfer both signals, in the same way similar to the conventional case. Also, in the last case, there is no need for frequency dividers to generate the $S$ signal, since it can be chosen as the clock signal itself.

\section{E. Communication procedure between two conventional $3 D$ routers}

To define a communication protocol for the TSVBOX-based $3 \mathrm{D}$ router, the conventional procedure should be explained first. The communication signalling between two conventional

\footnotetext{
${ }^{3}$ Such clock frequency division can be realized easily using injection-locked frequency dividers (ILFDs). Most of ILFDs have been optimized for division by even numbers [28].
}

3D routers inside the 3D NoC shown in Fig. 13, is done using the synchronous $R E Q$ and $A C K$ signals to transfer a flit from upstream to downstream router. This communication protocol can be summarized as follows:

(1) The transmitting router initiates a request to the receiving router by raising the $R E Q$ signal to ' 1 '. At the same time the data flit is ready on the data bus.

(2) After at least one cycle, the initiated request will be recognized by the input controller of the receiving router. If there is at least one free slot in the FIFO buffer, the packet will be read and the acknowledgement $A C K$ signal will be set to ' 1 ', announcing that the packet is received successfully.

(3) After another cycle from setting the $A C K$, the transmitting router will reset the $R E Q$ again to ' 0 ', upon detecting the assertion of $A C K$. Resetting $R E Q$ is the end of the communication operation.

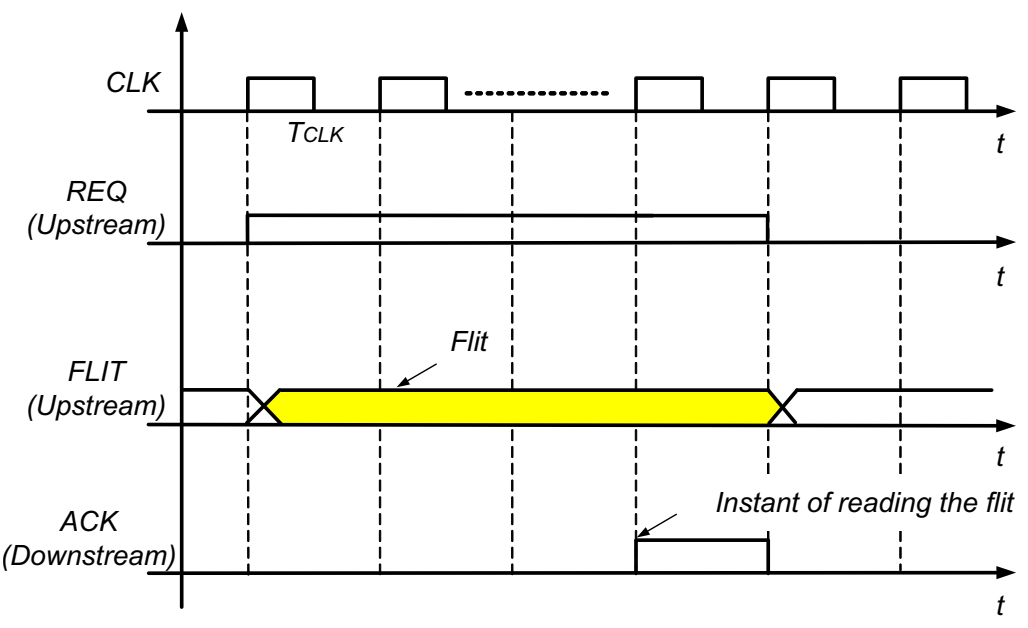

Fig. 13: Synchronous communication protocol.

\section{F. Communication procedure between two TSVBOX-based $3 D$ routers}

The communication protocol for the TSVBOX-based 3D routers, is the same as conventional procedure of the conventional routers for horizontal hops. For vertical hops the procedure is changed to account for extra cycles needed by the TSVBOX to transfer the data. As shown in Fig. 14, a new internal signal called OnePulse is introduced. OnePulse is used to power gate the driving circuit of the $S$ and $\bar{S}$ signals so as to reduce their power consumption when there is no transmission is in progress. This signal is activated when the output controller decides to send a flit to the downstream router. Since the TSVBOX needs $2 n . T_{C L K}$ to transfer two multiplexed signals successfully, the OnePulse signal lasts for only $2 n . T_{C L K}, n=0.5,1,2,3$, etc. as shown in Subsection VI-D Since both $S$ and $\bar{S}$ are needed only during transmission, the OnePulse signal can be used to activate or deactivate those two overhead signals. This activation or deactivation can be translated as power gating technique, as shown in Fig. 15 


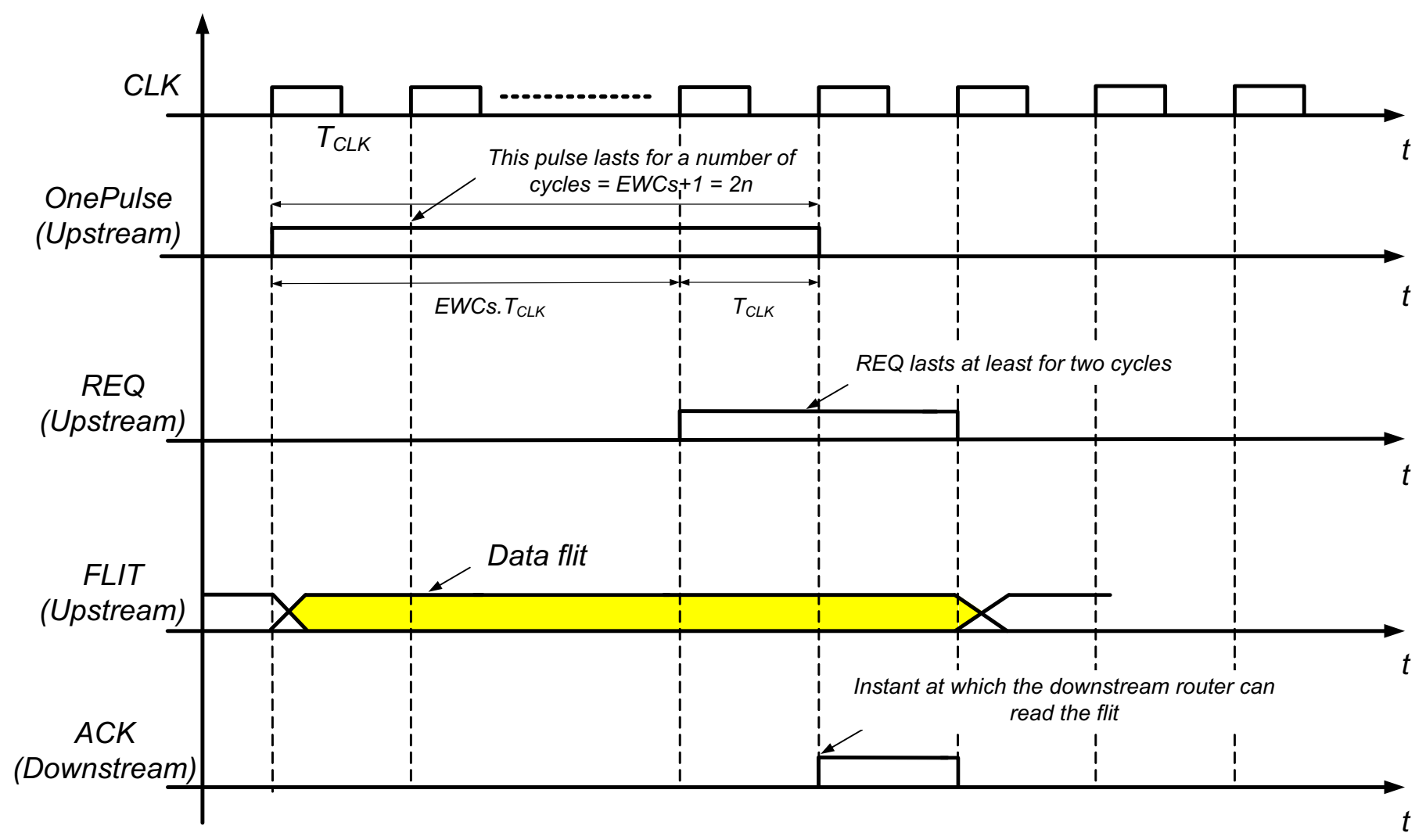

Fig. 14: Vertical communication synchronous protocol between two TSVBOX-based 3D routers.

Now the TSVBOX vertical communication procedure can be summarized as follows:

(1) The upstream router set the internal OnePulse signal that enables both $S$ and $\bar{S}$ signals, so that the two multiplexed signals can pass to the data bus first.

(2) Since the downstream router takes at least one cycle to read $R E Q$ after its initiation, $R E Q$ can be set by the upstream router after $(2 n-1) \cdot T_{C L K}$.

(3) Step 2 of the conventional procedure of Subsection VI-E

(4) Step 3 of the conventional procedure of Subsection VI-E

As observed the TSVBOX-based 3D routers imposes extra waiting cycles (EWCs) equal $(2 n-1)$ which is the number of $T_{C L K}$ cycles that the router must wait before initiating a request, and thus it may degrade the overall 3D NoC performance. However, we will show in Section VII-D that this performance degradation is dependent on the traffic pattern of the 3D NoC and other parameters as well.

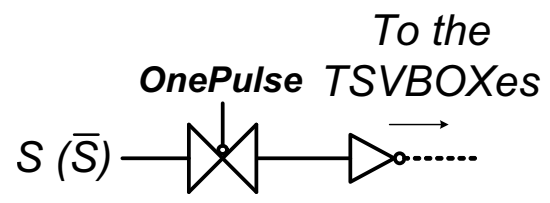

Fig. 15: Simple power gating technique using OnePulse signal.

\section{TSV MULTIPLEXING SCALABILITY ANALYSIS}

In the previous sections TSV multiplexing has been studied in details for $2 \times 1 \mathrm{TSV}$ multiplexing ratio. In this section the study is generalized for the general case of $N_{M U X} \times 1 \mathrm{TSV}$ multiplexing, where $N_{M U X}$ is the TSV multiplexing ratio $\left(N_{M U X} \geq 2\right) . N_{M U X}$ is assumed to be a power of 2 arbitrary integer, i.e. $N_{M U X}=2,4$, 8, etc..

Fig. 16 shows the TSVBOX SystemC-A circuit modeling for the general case of $N_{M U X} \times 1$ multiplexing ratio and a special case when $N_{M U X}=4$. The mechanism can be considered as a generalization for the previous mechanism of $2 \times 1$ multiplexing. As shown in Fig. 16a the multiplexed signals are passing through the TSVBOX during exclusive combination of the selection control signals. And since we have $2^{N_{M U X}}$ different combinations of the $S$ 's signals, i.e. $\left(S_{1} \cdot S_{2} \cdots S_{\log _{2} N_{M U X}}\right)$, $\left(S_{1} \cdot S_{2} \cdots \bar{S}_{\log _{2} N_{M U X}}\right), \cdots,\left(\overline{S_{1}} \cdot \overline{S_{2}} \cdots \bar{S}_{\log _{2} N_{M U X}}\right)$, each signal from the $N_{M U X}$ different input signals can pass exclusively through the TSVBOX. For example, in $4 \times 1$ multiplexing Figs. (16b16c), $V_{1}$ passes through the TSVBOX if all the $S$ 's signals are '1' $\left(S_{1} \cdot S_{2}=1\right)$, and this is only valid during the first half cycle of $S_{1}$, while $V_{2}$ passes if $S_{1} \cdot \overline{S_{2}}=1$, and this combination can be logic '1' only during the second half cycle of $S_{1}$. The same logic can be repeated for $V_{3}$ and $V_{4}$.

In the following subsections, the analysis of scalability study is provided to show how much higher TSV multiplexing ratios will impact control signals ( $S$ 's), EWCs, and TSVBOX delays. In the final subsection, the scalability impact on performance 
under different traffic patterns is analyzed.

\section{A. Effect of multiplexing on selection control signals}

As shown in Fig. 16a, the number of selection control signals ( $S$ 's) used is always $\log _{2}\left(N_{M U X}\right)$. However, we need to generate $N_{M U X}$ signals from these $\log _{2}\left(N_{M U X}\right)$ signals to drive the TSVBOX. This means that, we need $N_{M U X}$ extra TSVs to transfer them for each vertical bus width. This, of course, enforces a limitation on the multiplexing ratio to be used, especially if $N_{B W}$ is comparable to $N_{M U X}$. For example, if $N_{B W}=128$ and $N_{M U X}=128$, then no TSV saving will be gained from the TSVBOX. Another limitation comes from the generation circuit needed to generate the $S$ 's signals (AND gates and the inverters). The good point is that, this generation circuit is not repeated in each layer, we assume it is only placed in the first layer beside the clock dividers. We assume also that the area of the system circuit on the first die is extremely larger than the area overhead of the signal generation circuit, hence negligible contribution to the overall area.

The frequencies of the selection control signals are related to each other, e.g. in $4 \times 1$ case, $f_{S_{1}}=2 f_{S_{2}}$, which is simply synthesized using digital frequency dividers. The selection signal with maximum frequency $\left(f_{S_{1}}\right)$ is determined using the same way explained in Subsection VI-D according to the relation between $T_{C L K}$ and $\frac{T_{S_{1}-\min }}{2}$ (minimum time duration required to transmit any of the input multiplexed signals).

\section{B. Effect of multiplexing on EWCs}

Again the TSVBOX imposes extra delay in the shape of extra waiting cycles (EWCs), and these extra delay cycles depend on $T_{C L K}, \frac{T_{S_{1}}}{2}$, and the TSV multiplexing ratio $N_{M U X}$. To calculate EWCs in case $N_{M U X}>2$, we can generalize the $2 \times 1$ case of Subsection VI-F. Depending on the relation between $T_{C L K}$ and $\frac{T_{S_{1}-m i n}}{2}$, the EWCs simply can be shown to be (from Fig. 14):

$$
E W C s=\frac{N_{M U X} \cdot \frac{T_{S 1}}{2}}{T_{C L K}}-1
$$

and since $\frac{T_{S 1}}{2}=n . T_{C L K}$, therefore

$$
E W C s=n \cdot N_{M U X}-1
$$

$$
\text { where } n=\left\{\begin{array}{lr}
2,3, \text { etc. } & : T_{C L K}<\frac{T_{S_{1}-\min }}{2} \\
1 & : \frac{T_{S_{1}-m i n}}{2} \leq T_{C L K}<T_{S_{1}-\min } \\
\frac{1}{2} & : T_{C L K} \geq T_{S_{1}-\min }
\end{array}\right.
$$

\section{Effect of multiplexing ratio on TSVBOX delays}

The increase in TSVBOX delay is linear with $N_{M U X}$ multiplexing ratio. The delay of any multiplexed signal passes

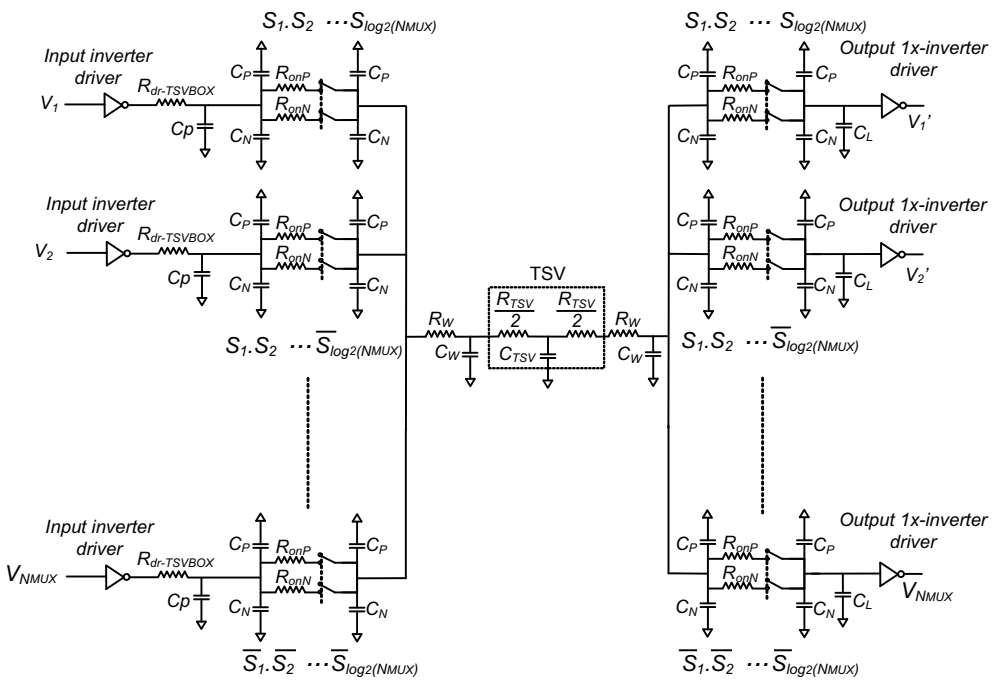

(a)
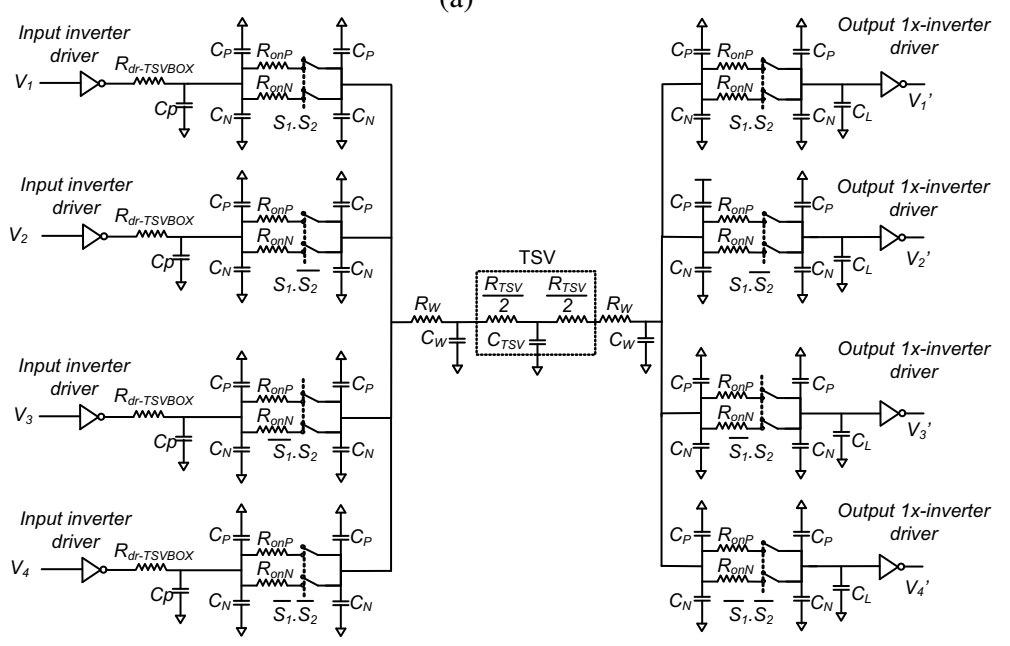

(b)

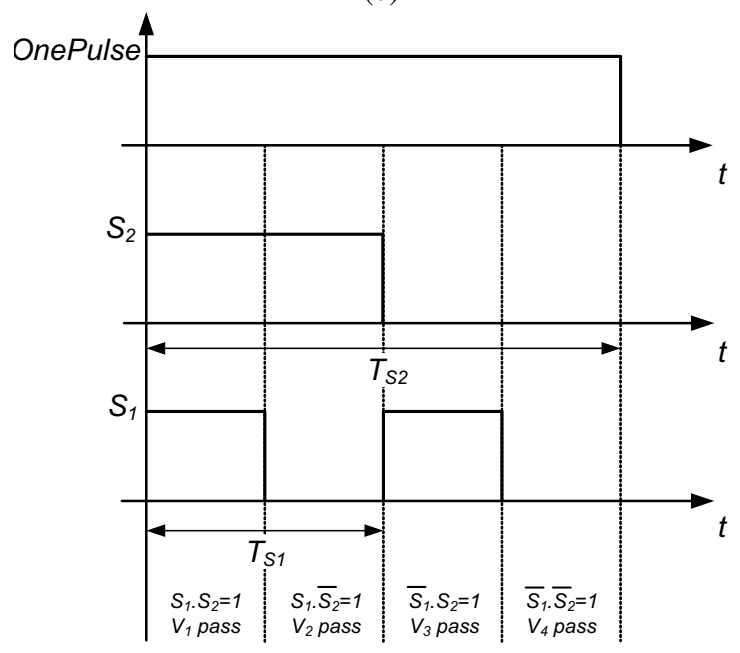

(c)

Fig. 16: TSVBOX circuit models for (a) the general case $N_{M U X} \times 1$ and special case in (b) $4 \times 1 \mathrm{TSV}$ multiplexing ratios. (C) Selection control signals required for $4 \times 1 \mathrm{TSV}$ multiplexing. $V_{1}$ passes when $S_{1} \cdot S_{2}=1, V_{2}$ passes when $S_{1} \cdot \overline{S_{2}}=1$, etc. 
through the TSVBOX can be calculated as

$$
\begin{gathered}
T_{d-T S V B O X}=\ln \frac{V_{D D}}{\left|V_{t h P}\right|-0.1\left(V_{D D}-\left|V_{t h P}\right|\right)} . \\
\left(R_{d r-T S V B O X} \cdot\left(C_{p}+C_{P N}\right)+\left(R_{d r-T S V B O X}+R_{P N}\right)\right. \\
\cdot\left(2 N_{M U X} \cdot C_{P N}+2 C_{W}+C_{T S V}\right)+ \\
\left.\left(R_{d r-T S V B O X}+2 R_{P N}\right) \cdot\left(C_{P N}+C_{L}\right)\right)
\end{gathered}
$$

where we considered the overlap penalty explained in Subsection VI-B As observed, Eq. 12 is a special case of Eq. 16 when $N_{M U X}=2$.

The multiplexing ratio affects the $S$ 's signals' delays as well, but this time, it is a positive effect; in other words, the higher the multiplexing ratio, the smaller the $S$ 's signals' delays. For example, for the case of $N_{M U X}=2$, each transmission gate contributes by $2 C_{g}$, hence the total loading contribution for either $S$ or $\bar{S}$ is $\left(4\left(\frac{N_{B W}}{N_{M U X}}\right) \cdot C_{g}=2 N_{B W} \cdot C_{g}=128 C_{g}\right)$, as depicted in Eq. 6 Another example, for the case of $N_{M U X}=4$, each TSVBOX contributes to each control signal $\left(S_{1} \cdot S_{2}, S_{1} \cdot \overline{S_{2}}\right.$, etc.) load by $2 C_{g}$ as well. However, since the total number of TSVBOXes has reduced to 16, i.e. $\frac{N_{B W}}{N_{M U X}}=\frac{64}{4}=16$, the total loading contribution would be $64 C_{g}$ for each control signal. In general, based on the previous analysis, Eq. 6 can be rewritten as

$$
\begin{aligned}
& T_{d-S}=\ln \frac{V_{D D}}{V_{D D}-\max \left(V_{t h N},\left|V_{t h P}\right|\right)} . \\
& \quad\left(R_{d r-S} \cdot\left(C_{p}+2 C_{W}+C_{T S V}+4 \frac{N_{B W}}{N_{M U X}} \cdot C_{g}\right)\right)
\end{aligned}
$$

\section{Effect of multiplexing ratio on on performance under different traffic patterns}

In this subsection we analyze the scalability issues on the performance of TSVBOX-based 3D NoC versus the conventional one. We choose three different traffic patterns; Matrix Transpose 4 . Uniform, and Hotspot. The packet injection process of each traffic flow is chosen to be a Poisson random process where the time interval between successive injections is represented as exponential random variable [29]. Poisson distributed injection rate is adopted in this study because it successfully characterizes the performance of multiprocessor applications [30, 31]. The transmitter waits for $T_{\text {interval }}=-\lambda \cdot \ln (U)$ between two successive packet transmissions, where $T_{\text {interval }}$ is the exponential random time interval between two successive transmitted packets, $\lambda$ is the reciprocal of the average injection rate of the process, i.e. the average waiting time between two successive transmissions, and $U$ is a uniform random variable between ' 0 ' and ' 1 ' [32]. Table III states all the definitions of the terms used in this analysis.

The following three equation 55 define the average extra waiting cycles $E W C s_{\text {avg }}$ per vertical hop faced by packets traverse vertically between 3D stack layers for Transpose,

\footnotetext{
${ }^{4}$ Henceforth, we would call it Transpose for simplicity.

${ }^{5}$ The proofs of these three equations are found in Appendix A.
}

Uniform, and Hotspot traffics, respectively.

$$
\begin{aligned}
& E W C s_{\text {avg-Transpose }}=2 \cdot N_{P K T} \cdot\left(n \cdot N_{M U X}-1\right) \cdot\left[\frac{N^{2}}{4}\right] \\
& E W C s_{\text {avg-Uniform }}=2 \cdot N_{P K T} \cdot\left(n \cdot N_{M U X}-1\right) \cdot \\
& {\left[\frac{1}{N} \cdot\left(\sum_{i=0}^{\frac{N}{2}-1} \frac{(N-i) \cdot(N-i-1)}{2}+\sum_{i=0}^{\frac{N}{2}-1} i \cdot\left(\frac{N}{2}-i\right)\right)\right]} \\
& E W C s_{\text {avg-Hotspot }}=2 \cdot N_{P K T} \cdot\left(n \cdot N_{M U X}-1\right) \cdot \\
& {\left[\frac{(1-h)}{N} \cdot\left(\sum_{i=0}^{\frac{N}{2}-1} \frac{(N-i) \cdot(N-i-1)}{2}+\sum_{i=0}^{\frac{N}{2}-1} i \cdot\left(\frac{N}{2}-i\right)\right)\right]}
\end{aligned}
$$

It can be seen that the difference between the above three equations lies in the terms between brackets "[]":

- In Transpose case $I=\frac{N^{2}}{4}$

- In Uniform case $I I=\frac{1}{N} \cdot\left[\sum_{i=0}^{\frac{N}{2}-1} \frac{(N-i) \cdot(N-i-1)}{2}+\right.$ $\left.\sum_{i=0}^{\frac{N}{2}-1} i \cdot\left(\frac{N}{2}-i\right)\right]$

- In Hotspot case $I I I=\frac{(1-h)}{N} \cdot\left[\sum_{i=0}^{\frac{N}{2}-1} \frac{(N-i) \cdot(N-i-1)}{2}+\right.$ $\left.\sum_{i=0}^{\frac{N}{2}-1} i .\left(\frac{N}{2}-i\right)\right]$.

Fig. 17 displays the variation of the previous three terms versus $N$, when $h=0.5$. We can notice that $I>I I>I I I$ for all values of $N$. Which means that Hotspot traffic is expected to show better performance than Uniform and Transpose traffics, and Uniform traffic in turn is expected to outperform Transpose.

\section{FABRICATION YIELD AND COST ANALYSIS}

In this section, fabrication yield and cost are analyzed based on the analysis of [1] for $\mathrm{W} 2 \mathrm{~W}$ bonding process. The design methodology proposed in our study in this paper introduces extra redundant hardware that may affect the overall system area and hence the overall yield and cost. Hence the analysis of this section is crucial for the overall evaluation of the TSV multiplexing technique. In the following subsections we briefly overview the normalized yield models derived in [1]. In the following analysis we use the terms defined in Table IV.

TABLE III: Definitions of all terms used in the performance analysis of the TSVBOX-based 3D NoC.

\begin{tabular}{|l|l|}
\hline Parameter & Definition \\
\hline$N$ & The number of 3D stack layers \\
\hline$N_{P K T}$ & The number of packets sent by each processing node \\
\hline$N_{M U X}$ & $\begin{array}{l}n=0.5,1,2,3,4, \text { etc. } \\
\text { dependent on the relation between } T_{C L K} \text { and } \frac{T_{S_{1}-m i n}}{2}\end{array}$ \\
\hline$n$ & $\begin{array}{l}0 \leq h \leq 1 . \\
\text { In Hotspot traffic, } h \text { represents the portion of the total } \\
\text { transmitted packets by any node that is directed to the } \\
\text { hotspot node in the 3D NoC }\end{array}$ \\
\hline$E W$ & $\begin{array}{l}\text { The average EWCs exposed by TSVBOX-based 3D NoC } \\
\text { per one vertical hop }\end{array}$ \\
\hline$X_{S I Z E}$ & The number of nodes in X dimension of the 3D NoC \\
\hline$Y_{S I Z E}$ & The number of nodes in Y dimension of the 3D NoC \\
\hline$Z_{S I Z E}$ & The number of nodes in Z dimension of the 3D NoC \\
\hline
\end{tabular}




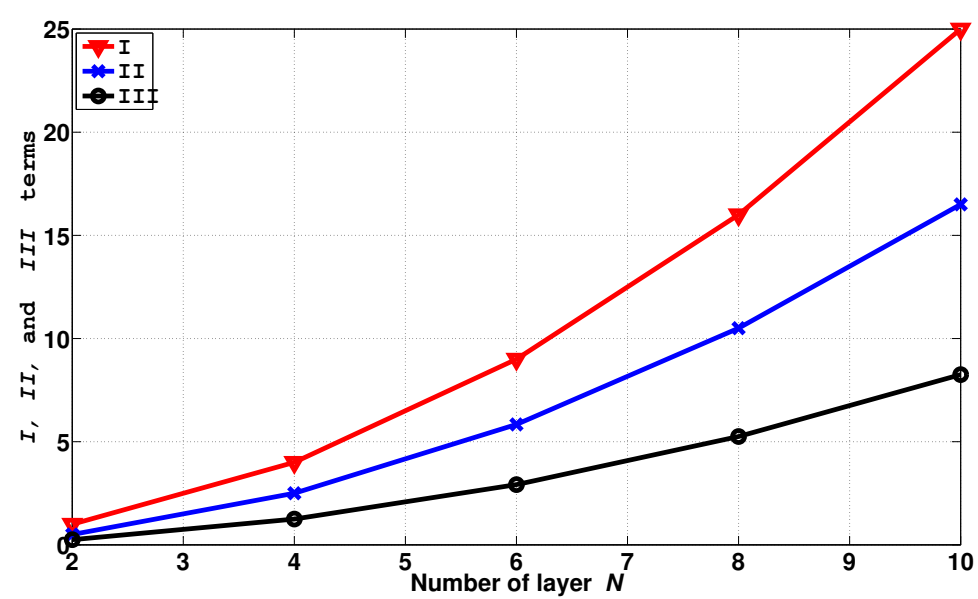

Fig. 17: The relation between the terms $I, I I$, and $I I I$.

\section{A. Fabrication yield analysis}

As stated in [1], there are two main components that affect the overall yield of the $\mathrm{W} 2 \mathrm{~W}$ bonding process:

- Normalized stacking yield: According to [1], the normalized stacking yield is equivalent to the normalized TSV yield $\left(Y_{T S V-n o r m}\right)$ which is the probability that all TSVs are non-faulty

$$
\begin{aligned}
Y_{S, W 2 W-\text { norm }} & =Y_{T S V-\text { norm }} \\
& =\frac{\left(1-f_{T S V}\right)^{\left(\left.N_{T S V}\right|_{\text {after multiplexing }}\right)}}{\left(1-f_{T S V}\right)^{\left(\left.N_{T S V}\right|_{\text {before multiplexing }}\right)}}
\end{aligned}
$$

where $\left.\quad N_{T S V}\right|_{\text {before multiplexing }}$ and $\left.N_{T S V}\right|_{\text {after multiplexing }}$ can be calculated as follows

$$
\left.N_{T S V}\right|_{\text {before }} \text { multiplexing }=2 M \cdot\left(N_{B W}+2\right)
$$

$$
\left.N_{T S V}\right|_{\text {after multiplexing }}=2 M \cdot\left(\frac{N_{B W}}{N_{M U X}}+2+N_{M U X}\right)
$$

Of course we can gain TSV yield improvement iff $\left.N_{T S V}\right|_{\text {after multiplexing }}<\left.N_{T S V}\right|_{\text {before multiplexing. }}$ If

TABLE IV: Definitions of all terms used in Eqs. 2131 .

\begin{tabular}{|l|l|}
\hline Parameter & Definition \\
\hline$\left.A_{\text {die }}\right|_{\text {before multiplexing }}$ & Die area before TSV multiplexing \\
\hline$\left.A_{\text {die }}\right|_{\text {after multiplexing }}$ & Die area after TSV multiplexing \\
\hline$A_{\text {routers,cores }}$ & $\begin{array}{l}\text { The aggregate area of NoC routers and cores } \\
\text { per one die }\end{array}$ \\
\hline $\mathrm{M}$ & Mesh size, e.g. M=4×4, $8 \times 8$, etc. \\
\hline$D_{T S V}$ & The TSV diameter \\
\hline$A_{T S V}$ & The cross-sectional area of one TSV \\
\hline$A_{M U X}$ & The multiplexer area of the TSVBOX \\
\hline$A_{d r-S}$ & The $S$ signal driver area \\
\hline$A_{T G}$ & $\begin{array}{l}\text { The transmission gate area used for clock gating } \\
\text { of the S's control signals }\end{array}$ \\
\hline$f_{T S V}$ & The probability of fabricating a non faulty TSV \\
\hline$N_{T S V}$ & $\begin{array}{l}\text { The number of TSVs between two layers in a } \\
\text { 3D stack }\end{array}$ \\
\hline$\alpha$ & $\begin{array}{l}\text { A constant depends upon the complexity of the } \\
\text { manufacturing process }\end{array}$ \\
\hline$D_{o}$ & Average density of defects per die. \\
\hline
\end{tabular}

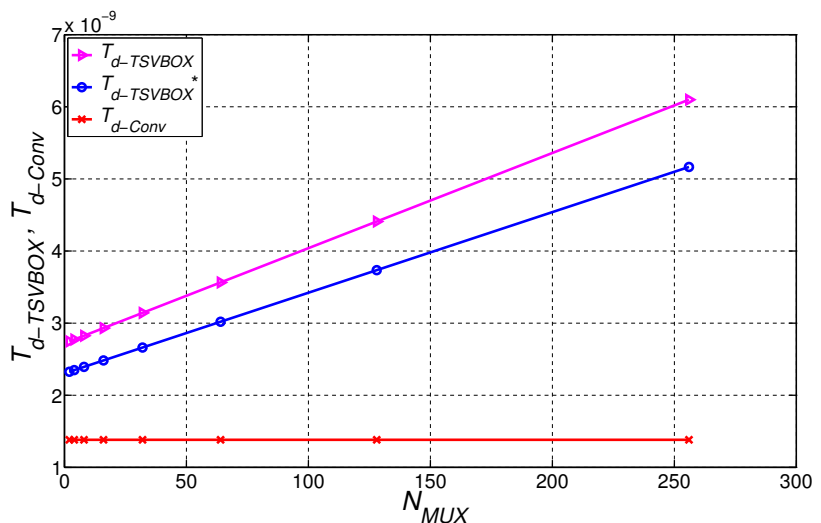

(a) $C_{T S V}=15 \mathrm{fF}$.

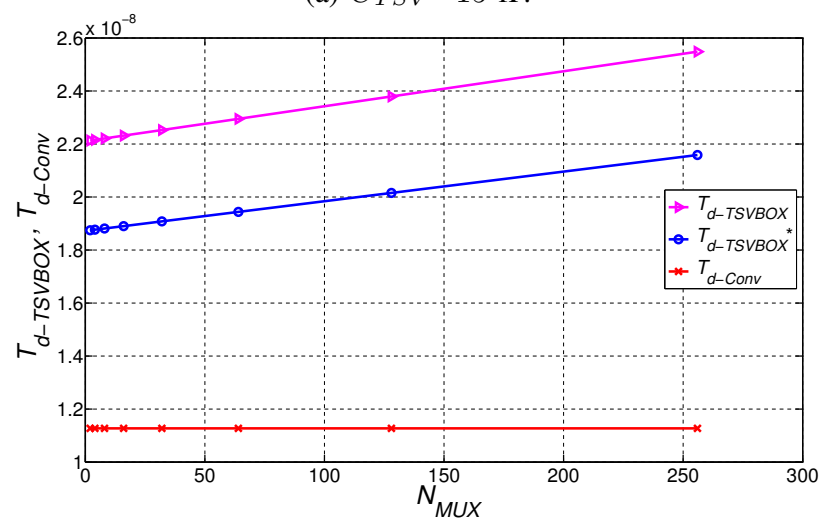

(b) $C_{T S V}=500 \mathrm{fF}$.

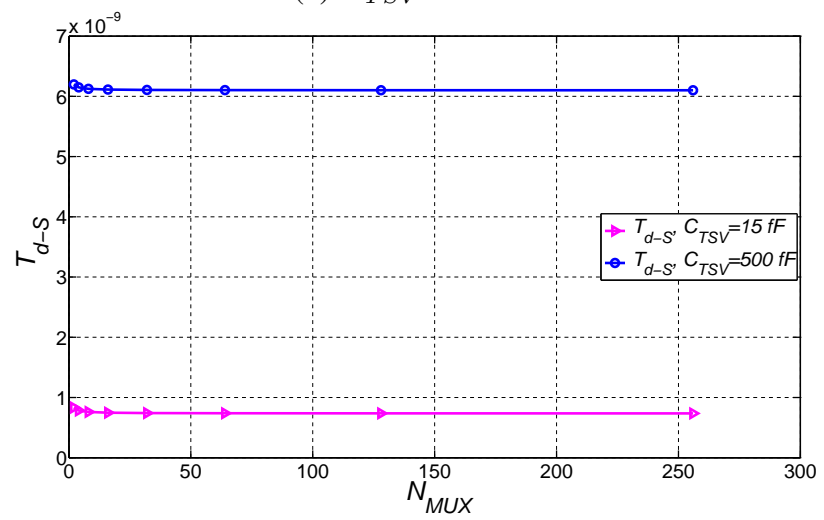

(c) Selection control signal delay.

Fig. 18: TSVBOX vs. conventional 3D interconnect delays for (a) $C_{T S V}=15 \mathrm{fF}$ and (b) $C_{T S V}=500 \mathrm{fF}$. The $T_{d-T S V B O X}{ }^{*}$ is a symbol for the TSVBOX delay of Eq. 5 before adding the $10 \%$ increase to $V_{i n H-m i n}$. In (c), the change of the selection control signal $T_{d-S}$ is depicted for $C_{T S V}=15 \mathrm{fF}$ and $C_{T S V}=500 \mathrm{fF}$.

we assume that both $N_{M U X}$ and $N_{B W}$ are power of 2 integers, then, we can show that the condition that makes $\left.N_{T S V}\right|_{\text {after multiplexing }}<\left.N_{T S V}\right|_{\text {before multiplexing }}$ is simply $N_{M U X} \leq \frac{N_{B W}}{2}$. For example, if $N_{B W}=256$ and $N_{M U X}=128$, so $\left.N_{T S V}\right|_{\text {before multiplexing }}=2 M \times(258)$ and $\left.\quad N_{T S V}\right|_{\text {after multiplexing }}=2 M \cdot(132) . \quad$ Now, 
for the same $N_{B W}$, if we assume $N_{M U X}=256$, $\left.N_{T S V}\right|_{\text {after multiplexing }}$ would be $2 M \times(259)$, which is clearly larger than 258; the required TSVs for no TSV multiplexing case.

- Die yield: Recalling [1], the normalized die yield $Y_{\text {die-norm }}$ can be represented as

$$
Y_{\text {die-norm }}=\left(\frac{\alpha+\left.D_{o} \cdot A_{\text {die }}\right|_{\text {before multiplexing }}}{\alpha+\left.D_{o} \cdot A_{\text {die }}\right|_{\text {after multiplexing }}}\right)^{\alpha}
$$

As observed, $Y_{\text {die-norm }}$ is in turn, dependent on the die area before and after TSV multiplexing, therefore it is affected by the proposed design methodology described in this paper. Regarding area, the proposed design methodology may affect the sizes of the drivers, especially for $S$ signal. Also, it requires special TSVs to transfer selection control signals between layers, which in turn adds extra area overhead for those extra TSVs. All these factors affect die area and in turn the die yield. Similar to the analysis of [1], the die area is dependent on die location, and the upper/bottom most dies have different areas than those of intermediate dies. The die area of upper and bottom-most dies $\left(\right.$ die $_{1}$, die $\left._{N}\right)$ before multiplexing can be estimated by

$$
\begin{array}{r}
\left.A_{\text {die } 1, N_{1}}\right|_{\text {before multiplexing }}=A_{\text {routers,cores }}+ \\
2 M \cdot\left(N_{B W}+2\right) \cdot A_{T S V}
\end{array}
$$

and for intermediate dies $\left(\mathrm{die}_{2}, \mathrm{die}_{3}, \ldots, \mathrm{die}_{N-1}\right)$

$$
\begin{array}{r}
A_{\text {die }_{2,3, \ldots,(N-1)} \mid \text { before multiplexing }}=A_{\text {routers }, \text { cores }}+ \\
4 M \cdot\left(N_{B W}+2\right) \cdot A_{T S V}
\end{array}
$$

Similar procedure can be followed for the TSVBOXbased 3D NoC case, therefore, for $d i e_{1}$ and $d i e_{N}$

$$
\begin{aligned}
& \left.A_{\text {die }_{1, N}}\right|_{\text {after multiplexing }}=A_{\text {routers,cores }}+ \\
& 2 M \cdot\left(\left(\frac{N_{B W}}{N_{M U X}}+2+N_{M U X}\right) \cdot A_{T S V}+N_{B W} \cdot A_{T G}\right. \\
& \left.\quad+N_{M U X} \cdot\left(A_{d r-S}+A_{T G}\right)\right)
\end{aligned}
$$

and for intermediate dies $\left(\operatorname{die}_{2}, \mathrm{die}_{3}, \ldots, \mathrm{die}_{N-1}\right)$

$$
\begin{array}{r}
A_{\text {die }_{2,3, \ldots,(N-1)} \mid \text { after multiplexing }}=A_{\text {routers,cores }}+ \\
4 M \cdot\left(\left(\frac{N_{B W}}{N_{M U X}}+2+N_{M U X}\right) \cdot A_{T S V}+N_{B W} \cdot A_{T G}\right. \\
\left.\quad+N_{M U X} \cdot\left(A_{d r-S}+A_{T G}\right)\right)
\end{array}
$$

where the TSV cross-sectional area $A_{T S V}$ can be calculated based on the value of TSV diameter $D_{T S V}$

$$
A_{T S V}=\pi\left(\frac{D_{T S V}}{2}\right)^{2}
$$

- Overall yield: Finally, the normalized overall yield $Y_{W 2 W-\text { norm }}$ is expressed according to [1] as:

$$
\begin{aligned}
& Y_{W 2 W-\text { norm }}=\left(Y_{\text {die-norm-top, bottom }}\right)^{2} \cdot \\
& \left(Y_{\text {die-norm-intermediate }}\right)^{(N-2)} \cdot\left(Y_{T S V-n o r m}\right)^{N-1}
\end{aligned}
$$

\section{B. Fabrication cost analysis}

As derived in [1], the overall W2W normalized cost $C_{W 2 W-\text { norm }}$ can be considered as the reciprocal of the overall W2W normalized yield

$$
\begin{aligned}
C_{W 2 W-\text { norm }} & =\frac{\left.C_{W 2 W}\right|_{\text {after multiplexing }}}{\left.C_{W 2 W}\right|_{\text {before }} \text { multiplexing }} \\
& =\frac{1}{Y_{W 2 W-\text { norm }}}
\end{aligned}
$$

\section{Simulation PlATFORM AND RESUlTS}

\section{A. $3 D$ signal path delays}

For the sake of fair comparison between the conventional and TSVBOX-based 3D NoCs, both are operated and designed based on the same input frequency and the same data driver size $\left(\left.K_{N}\right|_{\text {before multiplexing }}=\left.K_{N}\right|_{\text {after multiplexing }}\right)$.

Figs. $18 \mathrm{a} 18 \mathrm{~b}$ depicts the change in $T_{d-T S V B O X}$ vs. multiplexing ratio $N_{M U X}$ under two different values of $C_{T S V}$. While $T_{C o n v}$ remains constant, $T_{d-T S V B O X}$ increases linearly with increasing $N_{M U X}$, and this is applicable for both Eqs. 5[12 of the $T_{d-T S V B O X}$ delays. Fortunately, the linear increase is very small, because of the small slope;

$$
\begin{aligned}
\text { Slope } & =2 \ln \frac{V_{D D}}{V_{t h p}} \cdot C_{P N} \cdot\left(R_{d r-T S V B O X}+R_{P N}\right) \\
& \approx 0.011178 \text { nsec per unit change in } N_{M U X}
\end{aligned}
$$

An important notice here, is that the slope is independent of the TSV technology. Depending on Fig. (18a 18b) in case of 500 fF TSV capacitance, the increase in $T_{d-T S V B O X}$ delay when $N_{M U X}$ changes from 2 to 256 is $3.35 \mathrm{nsec}$, while for $15 \mathrm{fF}$ TSV capacitance it was exactly $3.35 \mathrm{nsec}$, as well. Apparently, those identical values support the theoretical expectation based on Eq. 32 .

Also Figs. 18a 18b depict that the TSVBOX shows a delay impact due to the redundant circuits added for multiplexing. Although, when $C_{T S V}$ is large, e.g. $C_{T S V}=500$ $\mathrm{fF}$, the ratio $\frac{T_{d-T S V B O X}}{T_{C o n v}}$ is slowly increasing by the increase of $N_{M U X}$, reducing the impact of $N_{M U X}$ on $T_{d-T S V B O X}$. For example, in case of $C_{T S V}=500 \mathrm{fF}$, for $N_{M U X}=2$, the ratio $\frac{T_{d-T S V B O X}}{T_{\text {Conv }}}=1.6631$, and for $N_{M U X}=256$ it is 1.9150 . However, for the case of $C_{T S V}=15 \mathrm{fF}$, the ratios were, 1.7108 and 3.7991 (more than the double of 1.7108), respectively.

Fig. 18c displays the change in $T_{d-S}$ versus $N_{M U X}$, and as stated before in Subsection VII-C the selection control signals show less delay for higher multiplexing ratios. However, the reduction is very small since the term $\left(4 \frac{N_{B W}}{N_{M U X}} \cdot C_{g}\right)$ of Eq. 17 is usually masked by the large values of $\left(C_{T S V}, 2 C_{W}\right)$ capacitors and because of the very small value of the gate capacitance $C_{g}$ itself. 
TABLE VI: Theoretical and simulational 3D signals' delays for conventional and TSVBOX paths for $65 \mathrm{~nm}$ technology for different TSV capacitances.

\begin{tabular}{|l|c|c|c|c|c|c|c|}
\hline \multirow{2}{*}{$\begin{array}{l}\text { 3D signal } \\
\text { delay }\end{array}$} & \multirow{2}{*}{ Unit } & \multicolumn{3}{|c|}{$15 \mathrm{fF}$} & \multicolumn{3}{c|}{$500 \mathrm{fF}$} \\
\cline { 3 - 8 } & & Theoretical & Simulational & $\mid$ error & Theoretical & Simulational & $\mid$ error $\mid$ \\
\hline$T_{d-C o n v}$ & $\mathrm{nsec}$ & 1.38 & 1.44 & $4.166 \%$ & 11.27 & 11.36 & $0.968 \%$ \\
\hline$T_{d-T S V B O X}$ & $\mathrm{nsec}$ & 2.33 & 2.31 & $0.87 \%$ & 18.75 & 18.74 & $0.053 \%$ \\
\hline$T_{d-S}$ & $\mathrm{nsec}$ & 0.833 & 0.9 & $7.44 \%$ & 6.198 & 6.42 & $3.46 \%$ \\
\hline
\end{tabular}

To check the accuracy of the delay models proposed, we compared the theoretical delays calculated from Eqs. $4|4| 12 \mid 6$, and the SystemC-A simulation delays. As depicted in Table $\mathrm{V}$, it is assumed that all the drivers are $1 \mathrm{x}$-inverters $\left(K_{N}=1\right.$, $K_{P}=2$ ), thus all the driver resistances are adjusted to their maximum values. As stated in Table $\mathrm{VI}$, the error between the theoretical and the simulational delay results does not exceed $7.5 \%$, which indicates the acceptable accuracy of Elmore-delay model.

\section{B. Performance comparison under synthetic traffic patterns}

The simulation results shown in Figs. (19/20/23) indicate the average delay and throughput comparisons of the two implemented $4 \times 4 \times 4$ 3D NoCs; the TSVBOX-based and convectional ones. The average delay of a packet is defined as the total cycles taken by the packet flits to cross the network towards its destination node. That delay spans from creation of the first flit (head flit) of the packet, to when its last flit (tail flit) is ejected at the destination (assuming immediate ejection), including source buffer queuing time in cycles [33]. For the average throughput, it is defined as the average ejection rate of the packets at their destination nodes. We set the simulation warm-up period to 2000 cycles in which we avoid calculating results until the network get congested [14]. Thereafter, similar to the methodology in [33], the simulation is run with 32,000 packets; 500 packet injected from each node, and the simulation continued at the prescribed packet injection rate till these packets have all been received, and their average delay and throughput are calculated.

Based on $T_{S-\min }$ we choose to run the simulations for three different clock periods to experience the effect of TSV multiplexing in different situations; $T_{C L K}=T_{S-\min }$, $T_{C L K}=\frac{T_{S-m i n}}{2}$, and $T_{C L K}=\frac{T_{S-\min }}{4}$. We choose to calculate $T_{S-\min }$ for $C_{T S V}=500 \mathrm{fF}$, since it will be an upper bound for the TSVBOX delay degradation. Though, the same conclusions derived later from Figs. $19|20| 23)$ will not change

TABLE V: Simulation setup for the sizes of the inverter drivers and their equivalent $O N$ resistances.

\begin{tabular}{|l|c|c|c|}
\hline Design parameter & \multirow{2}{*}{ Unit } & \multicolumn{2}{|c|}{$65 \mathrm{~nm}$} \\
\cline { 3 - 4 } & & $15 \mathrm{fF}$ & $500 \mathrm{fF}$ \\
\hline$\left(K_{N-C o n v}, K_{P-C o n v}\right)$ & - & $(1,2)$ & $(1,2)$ \\
\hline$\left(K_{N-T S V B O X}, K_{P-T S V B O X}\right)$ & - & $(1,2)$ & $(1,2)$ \\
\hline$\left(K_{N-S}, K_{P-S}\right)$ & - & $(1,2)$ & $(1,2)$ \\
\hline$R_{d r-C o n v}$ & $\mathrm{k} \Omega$ & 21.654 & 21.654 \\
\hline$R_{d r-T S V B O X}$ & $\mathrm{k} \Omega$ & 21.654 & 21.654 \\
\hline$R_{d r-S}$ & $\mathrm{k} \Omega$ & 21.654 & 21.654 \\
\hline
\end{tabular}

TABLE VII: Different delays for different multiplexing ratios.

\begin{tabular}{|l|c|c|c|}
\hline \multirow{2}{*}{ Delays } & \multicolumn{3}{|c|}{$N_{M U X} \times 1$} \\
\cline { 2 - 4 } & $2 \times 1$ & $4 \times 1$ & $16 \times 1$ \\
\hline$T_{d-T S V B O X}$ & 19.06 & 19.08 & 19.22 \\
\hline$T_{S-\min }$ & 24.40 & 24.42 & 24.55 \\
\hline
\end{tabular}

for the case of $C_{T S V}=15 \mathrm{fF}$. Since $T_{d-T S V B O X}$ is multiplexing ratio dependent, Table VII displays the values of the $T_{d-T S V B O X}$ and $T_{d-S}$ versus $N_{M U X}$.

As discussed in Subsection VII-B, depending on the relation between $T_{C L K}$ and $T_{S-m i n}$, TSVBOX adds EWCs, which are calculated using Eq. 15 and depicted in Table VIII for different multiplexing ratios.

What has been proven analytically in Subsection VII-D can be deduced clearly from Figs. 19, 20,23. We prove previously in Subsection VII-D that degradation in performance due to EWCs of the design methodology is application dependent, and we show analytically that Hotspot traffic gives closer performance to the conventional case than other traffic patterns. We show also that the performance under Transpose traffic will be the worst against other patterns; Hotspot and Uniform. Those conclusions are very readable from all performance figures.

As expected, for the less $T_{C L K}$ compared to $T_{S-\min }$ the degradation in performance metrics will be more. For example when $T_{C L K}=T_{S-\text { min }}$ in Figs. $19 \mathrm{c}$ and $19 \mathrm{f}$, saturation point occurs at Injection rate $(I R) \approx 0.01$ packet per cycle for $16 \times 1$ multiplexing, while for the conventional case it is around $\approx 0.05$ packet per cycle. This situation is repeated in all cases but it is decreased or worsen according to the traffic pattern used or the relation between $T_{C L K}$ and $T_{S-\min }$.

In Figs. 19a|19d/20a/20d/21a/21d we observe that the TSVBOX does not degrade the performance for $2 \times 1$ multiplexing. This strengthens the multiplexing technique since it can improve the yield without degrading the performance.

In summary, though the TSVBOX increases the delay of the $3 \mathrm{D}$ signals, according to the application and the relation between $T_{C L K}$ and $T_{S-m i n}$, the TSVBOX-based 3D NoC can mitigate the performance degradation and shows a comparable performance compared to conventional 3D NoC even for high multiplexing ratios.

\section{Performance comparison using real Benchmark traffic}

In this subsection another performance comparison is shown, this time under real benchmark traffic of the well known dVOPD video application traffic [37]-[40] with task graph shown in Fig. 22. Since mapping the tasks of the 


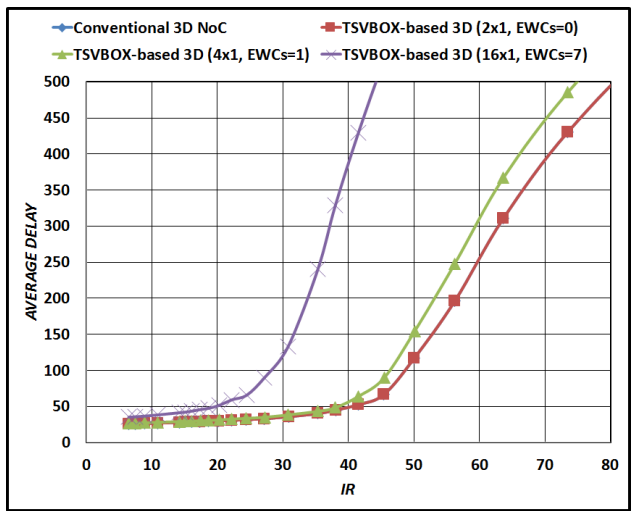

(a) $T_{C L K}=T_{S-\min }$

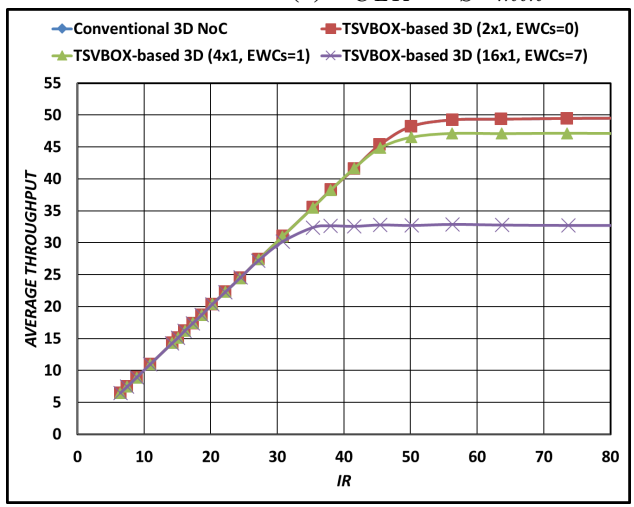

(d) $T_{C L K}=T_{S-\min }$

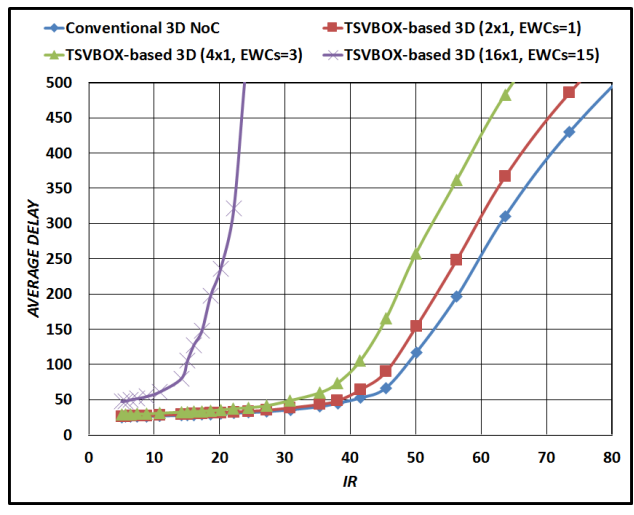

(b) $T_{C L K}=0.5 T_{S-\min }$

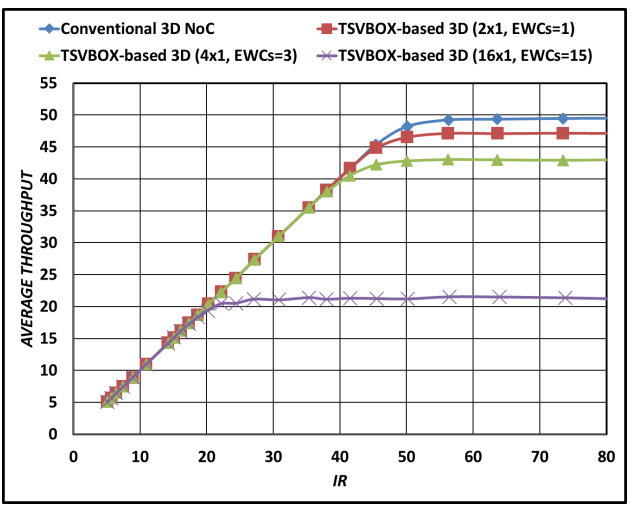

(e) $T_{C L K}=0.5 T_{S-\min }$

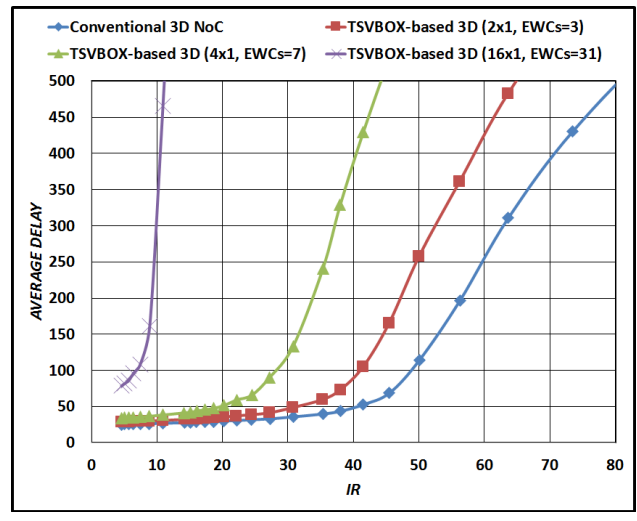

(c) $T_{C L K}=0.25 T_{S-\min }$

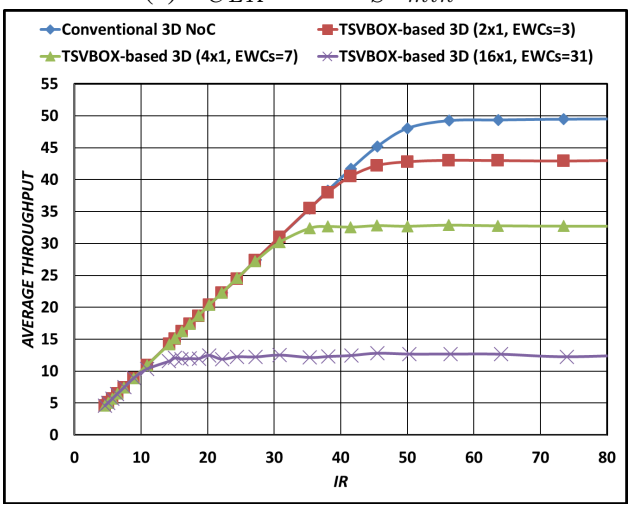

(f) $T_{C L K}=0.25 T_{S-\min }$

Fig. 19: Average delay and throughput under Transpose traffic pattern.

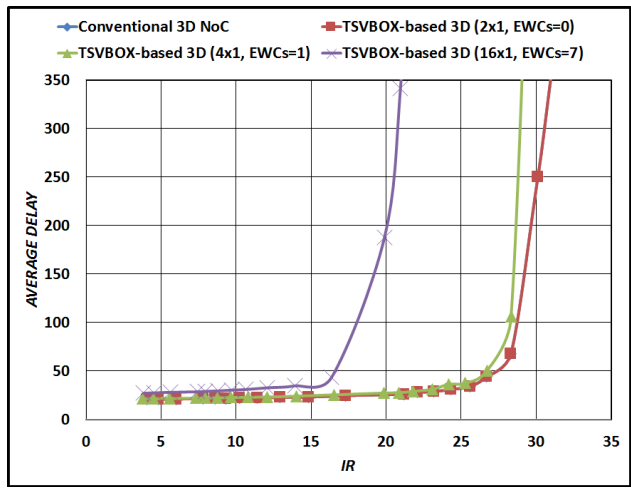

(a) $T_{C L K}=T_{S-\min }$

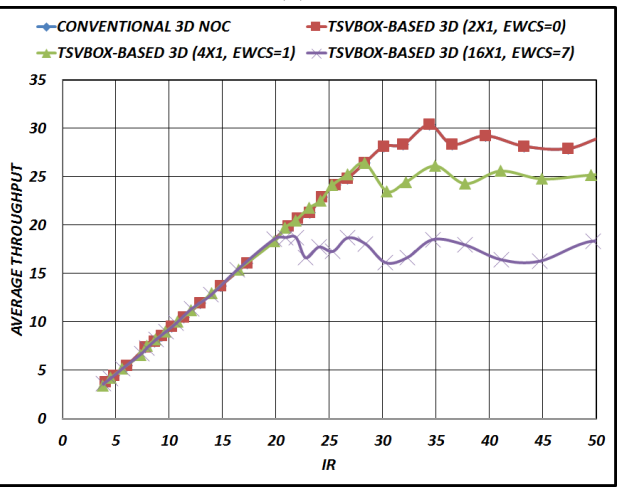

(d) $T_{C L K}=T_{S-\min }$

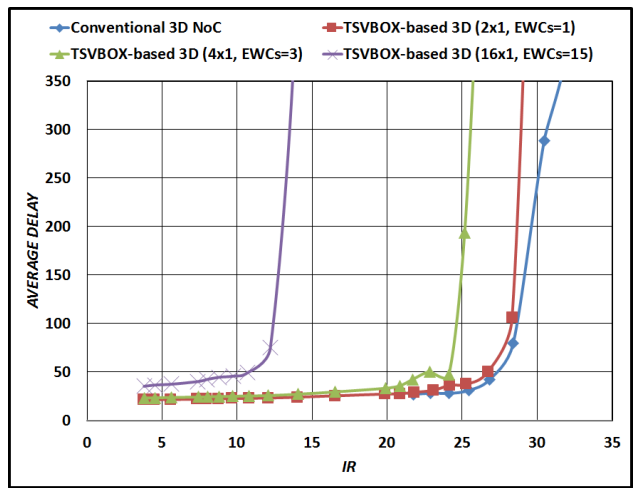

(b) $T_{C L K}=0.5 T_{S-\min }$

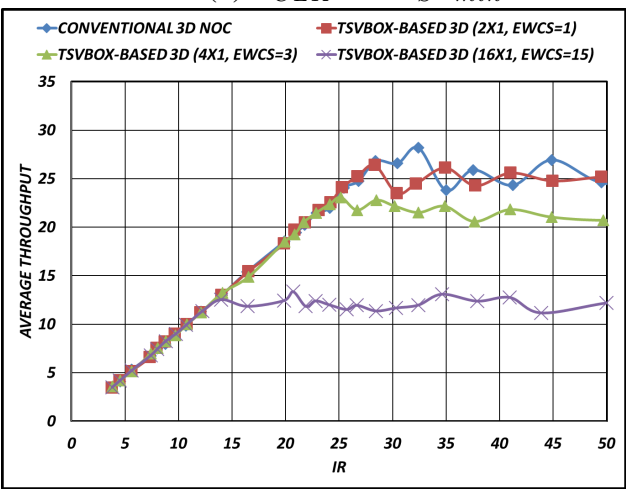

(e) $T_{C L K}=0.5 T_{S-\min }$

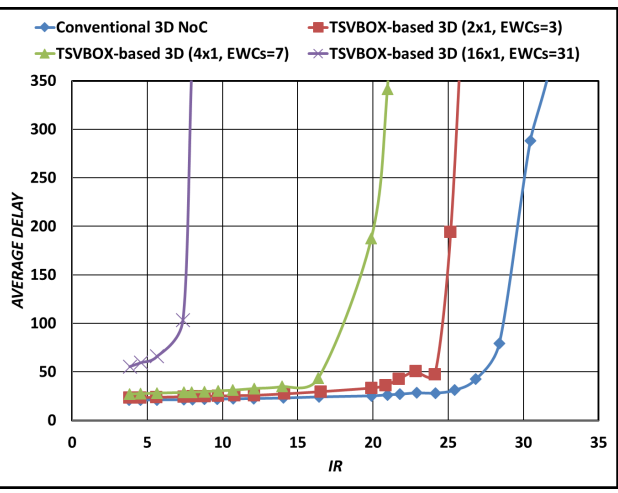

(c) $T_{C L K}=0.25 T_{S-\min }$

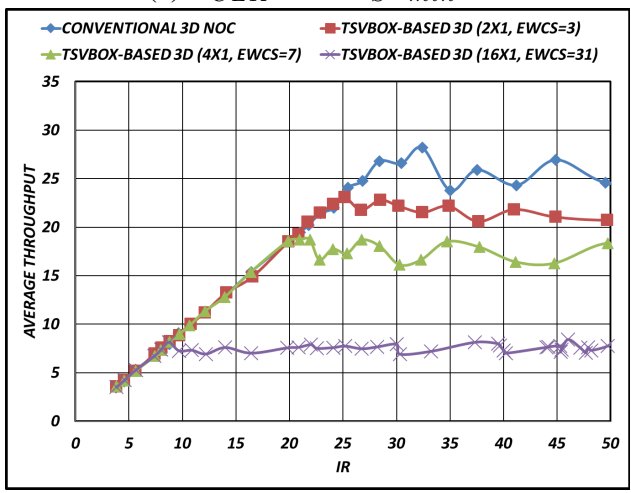

(f) $T_{C L K}=0.25 T_{S-\min }$

Fig. 20: Average delay and throughput under Uniform traffic pattern. 


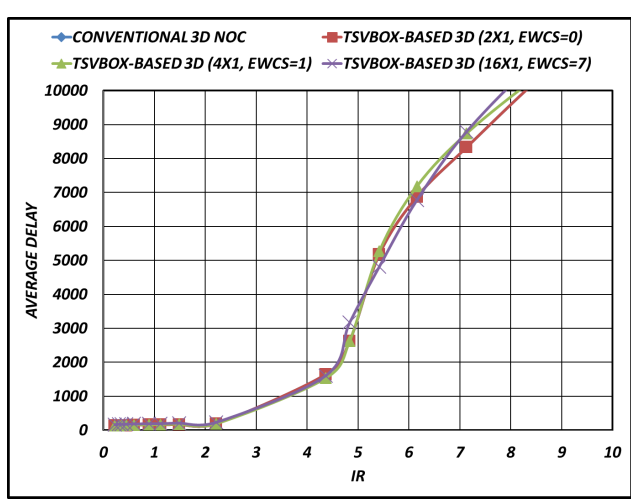

(a) $T_{C L K}=T_{S-\min }$

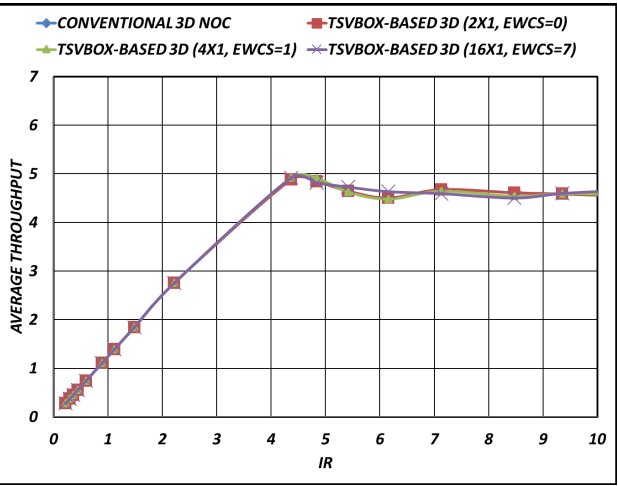

(d) $T_{C L K}=T_{S-\min }$

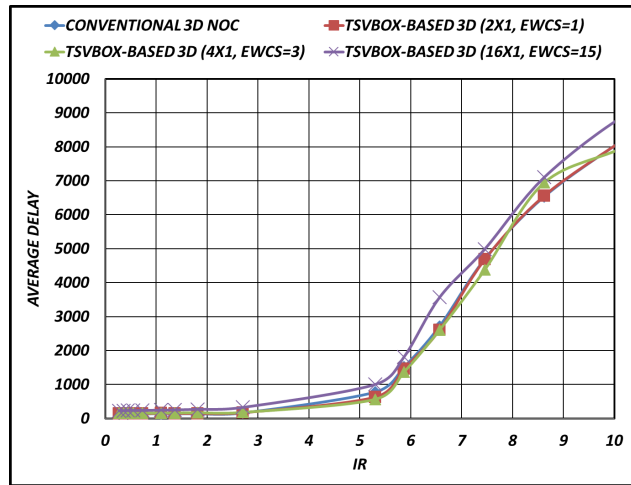

(b) $T_{C L K}=0.5 T_{S-\min }$

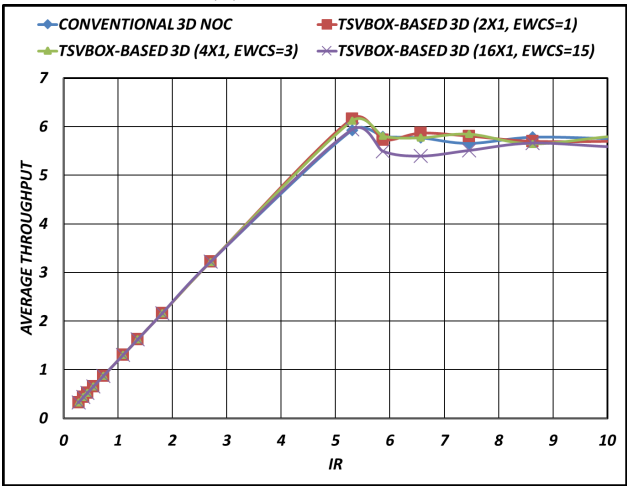

(e) $T_{C L K}=0.5 T_{S-\min }$

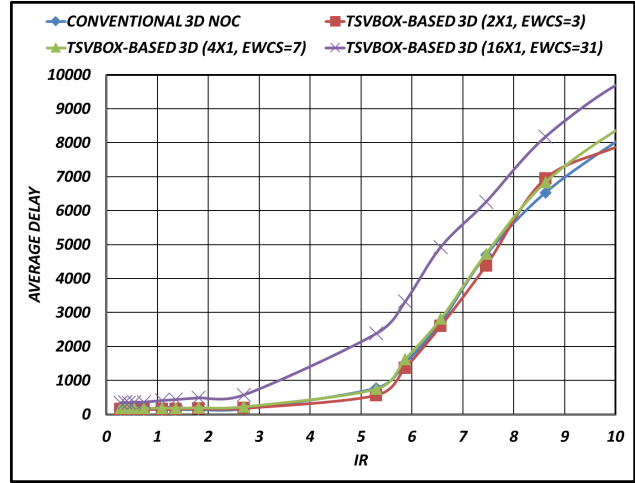

(c) $T_{C L K}=0.25 T_{S-\min }$

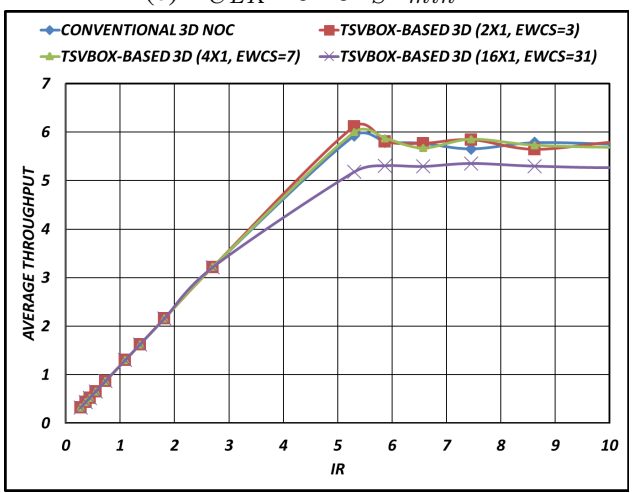

(f) $T_{C L K}=0.25 T_{S-\min }$

Fig. 21: Average delay and throughput under Hotspot traffic pattern.

TABLE VIII: EWCs under different clock periods and multiplexing ratios.

\begin{tabular}{|l|c|c|c|}
\hline \multirow{2}{*}{$T_{C L K}$} & \multicolumn{3}{|c|}{ EWCs } \\
\cline { 2 - 4 } & $2 \times 1$ & $4 \times 1$ & $16 \times 1$ \\
\hline$T_{S-\min }$ & 0 & 1 & 7 \\
\hline$\frac{T_{S-\min }}{2}$ & 1 & 3 & 15 \\
\hline$\frac{T_{S-\min }}{4}$ & 3 & 7 & 31 \\
\hline
\end{tabular}

task graph to the NoC cores is NP hard problem [37]-[40], we model this mapping problem using MiniZinc modeling discrete optimization language [42]-[43]. Modeling is done to get the mapping with with minimum communication cost, where the communication cost is defined as the sum of the communication bandwidth between each two tasks or vertices in the task graph $\left(B W_{i j}\right)$ multiplied by the number of hops between those two tasks in the $\mathrm{NoC}\left(H_{i j}\right)$. Thus the objective function can be represented as

$$
\operatorname{minimize} \sum_{i} \sum_{j} B W_{i j} \cdot H_{i j}
$$

The difference in performance between TSVBOX-based and conventional 3D NoCs arises from the extra hops the TSVBOX may add to the flits going vertically because of the extra waiting cycles. Our implemented simulation platform introduces a 9 cycles delay per one horizontal or vertical hop for conventional 3D NoC while for TSVBOX-based 3D NoC it gives the same horizontal hop delay of 9 cycles but $9+$ EWCs per vertical hop. a new metric is introduced to account for this vertical delay which is VHD (Vertical Hop Delay). Of course VHD is constant equal 9 for conventional 3D NoC while it is 9+EWCs for TSVBOX-based 3D NoC.

Similar conclusions to the ones we got in the previous subsection are made from Figs. 23a and 23b, again the performance of the TSVBOX is dependent on the EWCs it introduces. For example if $\mathrm{VHD}=9(\mathrm{EWCs}=0)$ we get similar performance to original conventional case without multiplexing and then the performance degrades based on the amount of EWCs being added.

\section{Performance comparison with TSV serialization technique}

Serialization is the most famous technique to reduce TSVs to increase the yield and reduce the fabrication cost. In this subsection we study the performance of the TSVBOX against the serialization technique. The common impact of both techniques is that they add extra delay to the 3D signals passes the vertical or interlayer interconnects. The EWCs delay added by the serialization is constant equal $N_{S E R}+2$ cycles, where $N_{S E R}$ is the serialization ratio, e.g. for $2 \times 1$ serialization $N_{S E R}$ is 2 . Though, the TSVBOX extra delay cycles is dependent on the frequency of operation and given by Eq. 15 In Eq. 15 the parameter $n$ is dependent on the relation between the TSVBOX delay and the operational clock. This leads to the fact that the EWCs of the TSVBOX can be less than the one of the serialization and the TSVBOX 


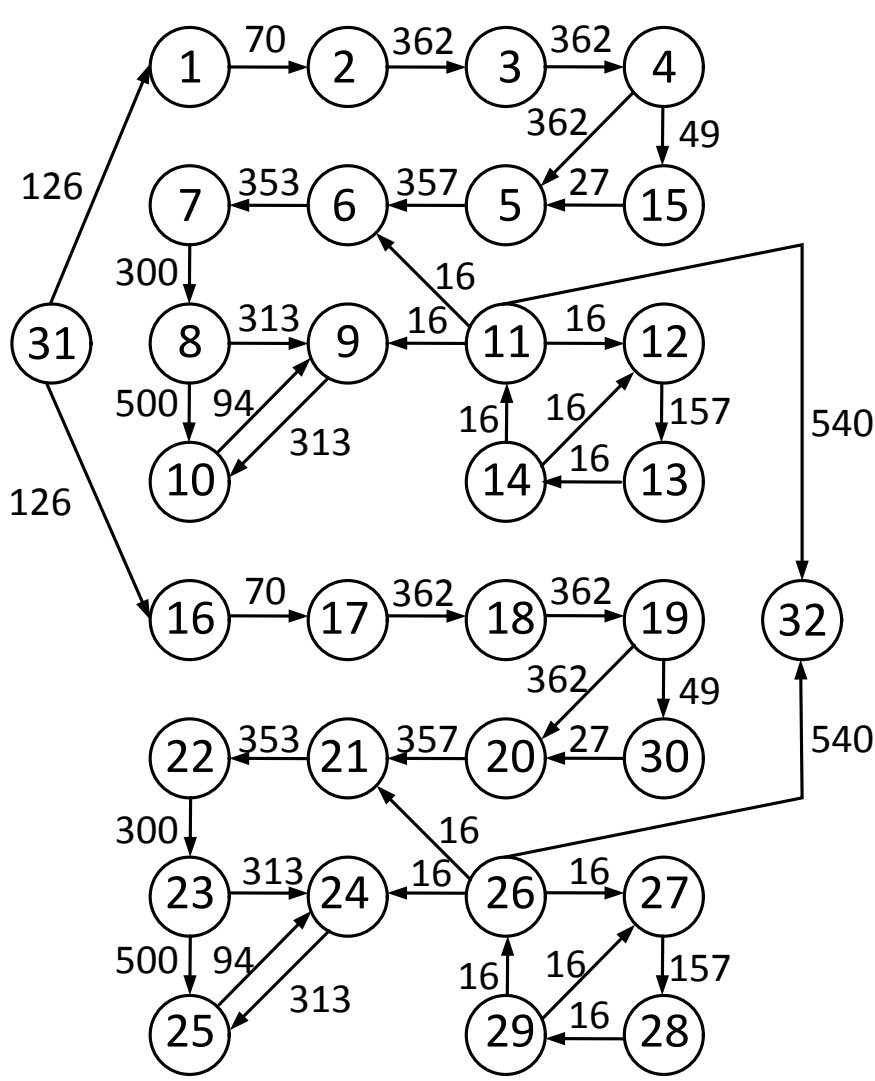

Fig. 22: dVOPD communication task graph with communication bandwidth stated in $\mathrm{MB} / \mathrm{s}$ on each edge [41] between each two tasks (vertices).

can show better performance than the serialization technique. Another advantage for the TSVBOX is that it performs better as the technology scales because we expect lower TSVBOX delay and thus lower $n$. The latter advantage gives more opportunities for the TSVBOX to be the best candidate to reduce the TSVs for future 3D chips.

Fig. 24 clarify those observations regarding performance difference between serialization and TSVBOX techniques. For example, in Figs. 24a and 24d for 2x1 multiplexing/serialization while serialization technique always adds 4 cycles delay $(\mathrm{EWCs}=4)$ the TSVBOX EWCs delays range between 0 and 3 cycles (refer to table VIII) so in all those cases the TSVBOX outperforms the serialization technique. For the other examples in Figs. (24b $24 \mathrm{e} \mid 24 \mathrm{c} / 24 \mathrm{f})$ and depending on the EWCs that each technique adds the TSVBOX outperforms the serialization in some situations and vice versa, e.g. for $4 \times 1$ multiplexing/serialization the TSVBOX outperforms the serialization when EWCs of the TSVBOX less than 6 which is the EWCs added by serialization and same conclusion can be concluded for $16 \times 1$ multiplexing/serialization.

\section{E. Power comparison}

Since there is no general benchmark method defined for NoCs to measure power consumption [34], we follow the methodology mentioned in [16, 30] to calculate the power

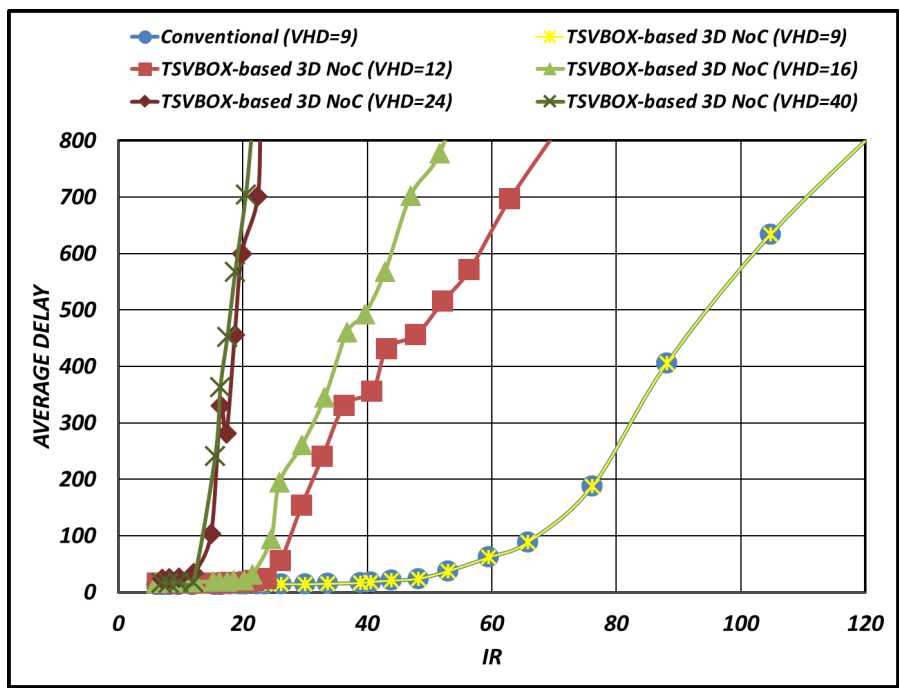

(a) Delay.

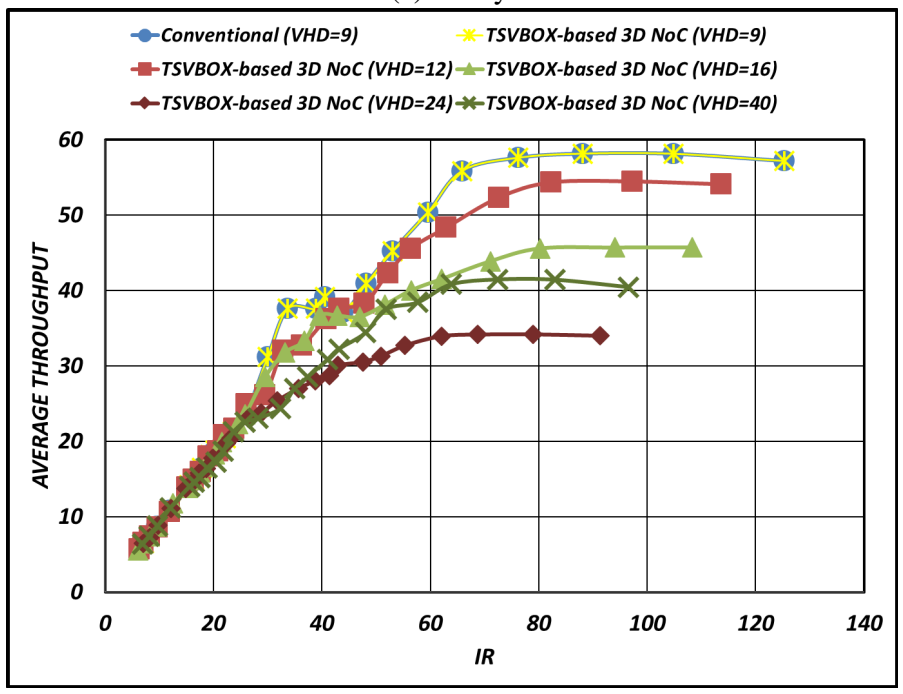

(b) Throughput.

Fig. 23: Average delay and throughput under real dVOPD benchmark traffic.

consumption of all power components: the intralayer interconnects, i.e. the 2D interconnects or links between routers located in the same layer of the 3D stack, the interlayer interconnects, i.e. the 3D interconnects or links between routers of two neighbor layers of the 3D stack, and the 3D routers themselves.

For both 3D and 2D interconnects, we developed a specific methodology based on the ability of SystemC-A to measure the currents flow through the modeling circuits of Figs. 616a for different input data. Large sequence of random flits are continuously fed to the 2D or 3D interconnects, at the same time the simulator takes samples of the currents of the interconnects every specified period of time and a counter is continuously counting the number of samples. After receiving all the sent flits, the simulator multiply the sum of the samples by $V_{D D}$ and take the average by dividing the calculated value by the counter value. 


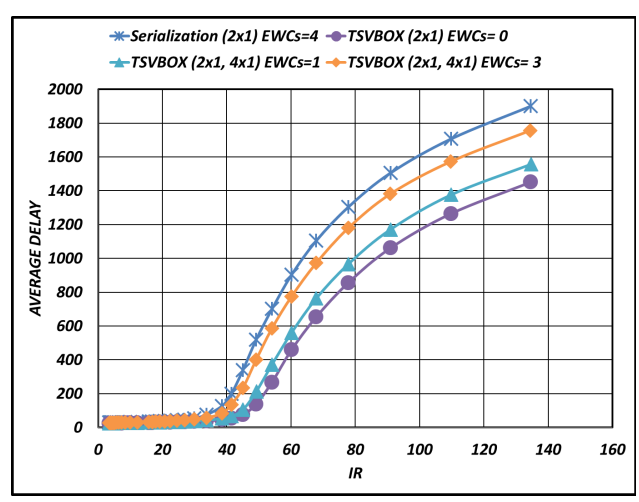

(a) 2x1 Multiplexing/Serialization.

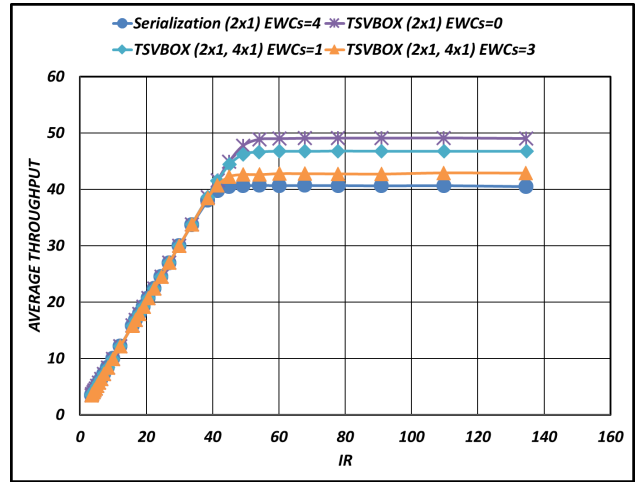

(d) 2x1 Multiplexing/Serialization.

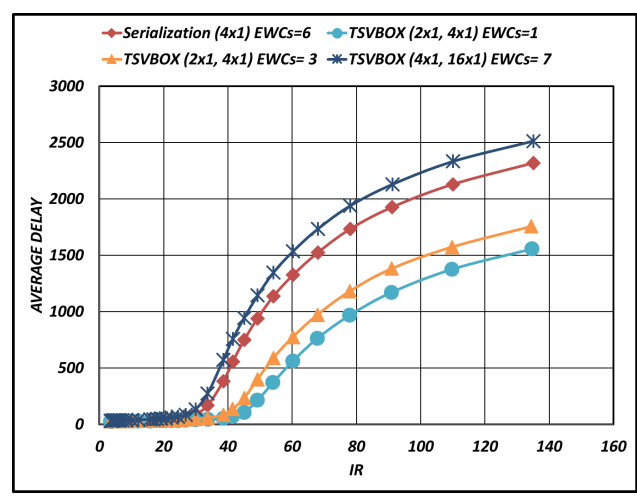

(b) $4 \times 1$ Multiplexing/Serialization.

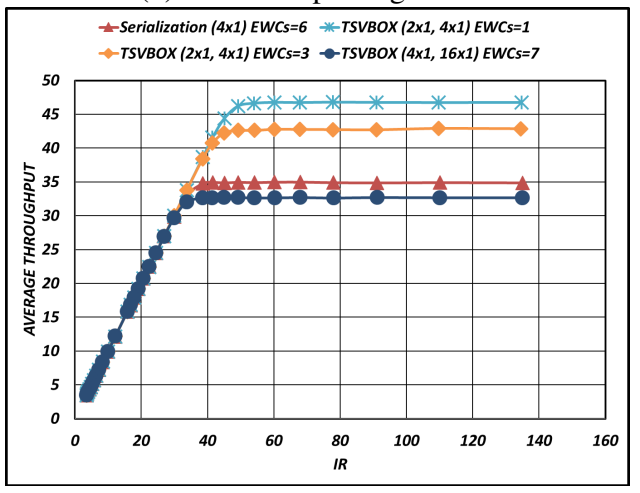

(e) $4 \times 1$ Multiplexing/Serialization.

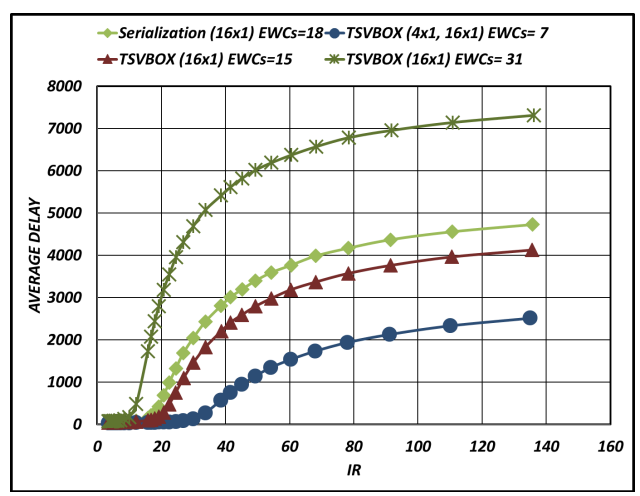

(c) 16x1 Multiplexing/Serialization.

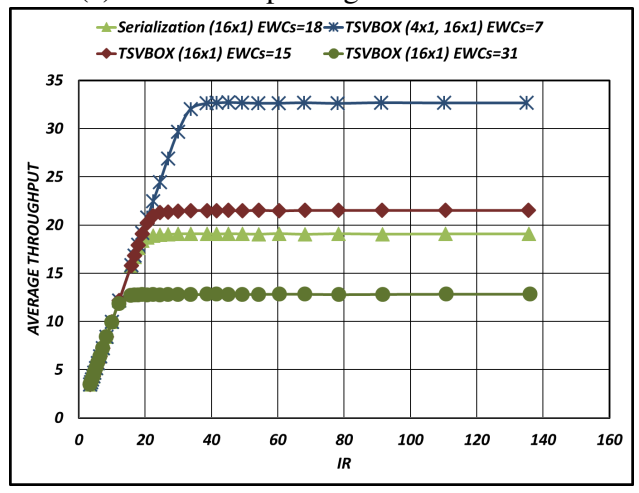

(f) $16 \times 1$ Multiplexing/Serialization.

Fig. 24: Performance comparison between TSVBOX and Serialization techniques for different TSV Multiplexing/Serialization ratios.

Similarly to the simulation scenario performed in SystemCA, we developed a model to measure the power consumption of the 3D router when flits pass through. Former, 3D router is written in Register Transfer Level (RTL) using Verilog language. Then, a switching activities scenario is created by injects a long random sequence of flits into the input ports of the 3D router. Afterwards, this scenario is applied on Modelsim to generate the VCD file. Then VCD file is translated to SAIF file. Later, the 3D router is synthesized by Design Complier (DC) from Synopsys and switching activities scenario is applied using the SAIF file. The synthesis is performed on $65 \mathrm{~nm}$ technology.

Power consumption results are shown in Fig. 25 As noticed for high TSV capacitance $\left(C_{T S V}=500 \mathrm{fF}\right.$, Figs. 25a 25b 25c ), the difference in power consumption is clearly against the TSVBOX. But the situation becomes much better for small TSV capacitance $\left(C_{T S V}=15 \mathrm{fF}\right.$, Figs. 25d 25e 25f $)$ because the $2 \mathrm{D}$ interconnects power and router power components are not affected by the reduction in TSV capacitance, and only the 3D interconnects power component does.

The trend in TSV fabrication technology is to decrease its dimensions and hence its capacitance as well. Therefore, and based on the previous results, the TSVBOX is adaptable to recent TSV technologies and its power consumption behavior will enhance more and more for future smaller TSVs.

Each of the various low-power-coding techniques [35] that geared towards minimizing the number of transitions and hence power consumption can be applied here. We expect that applying such technique will reduce the power consumption of the TSVBOX more and makes it too close or even lower in consumption than the conventional case. This is due to the fact that the coupling parasitics (capcitance and inductance) in the TSVBOX-based 3D NoCs would be less than the conventional case because the reduction in TSV count results in more space between TSVs. But for simplicity sake, no professional coding techniques is considered in our analysis and we dedicate it as a future work.

\section{F. Yield enhancement and cost reduction versus data bus width and number of $3 D$ stack layers}

To know the TSVBOX effect on die yield, the parameters of Table IV should be known first. As explored in [15], microprocessor-like dies are usually large. Sticking to the assumptions stated in [15], A $A_{\text {routers,cores }}$ is assumed to be $100 \mathrm{~mm}^{2}$ for $\mathrm{M}=4 \times 4$. Also we assume that $A_{\text {routers,cores }}$ has direct dependent on mesh size $\mathrm{M}$, i.e. for $\mathrm{M}=8 \times 8$, $A_{\text {routers, cores }}=\frac{100 \mathrm{~mm}^{2} \times(8 \times 8)}{4 \times 4}$, etc.. The number of layers is selected to be in the range from 2 to 8 layers. TSV diameter is assumed $1 \mu \mathrm{m}$ to match the ITRS trends for TSV technology.

In [1], MUX area was $16 \mu \mathrm{m}^{2}$ for $180 \mathrm{~nm}$ technology, so in this paper we estimate the MUX area for $65 \mathrm{~nm}$ using ITRS suggested scaling factor of 0.7 , thus the MUX area would be $16 \times(0.7)^{3} \approx 5.5 \mu \mathrm{m}^{2}$. Since the MUX circuit is composed of two transmission gates and some small connection wires, the transmission gate area can be assumed half the area of the MUX; $A_{T G} \approx 2.75 \mu \mathrm{m}^{2}$. Regarding driver area, we depend on 


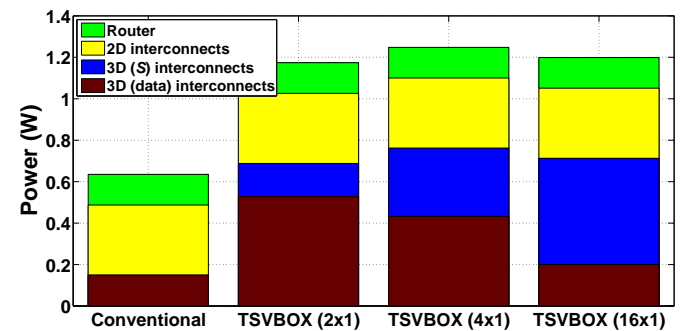

(a) Transpose $\left(C_{T S V}=500 \mathrm{fF}\right)$.

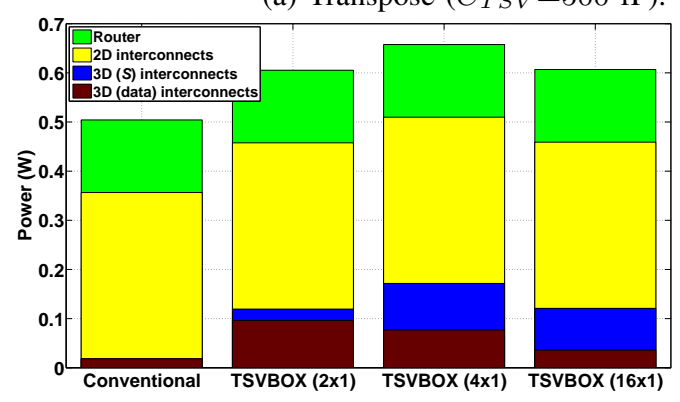

(d) Transpose $\left(C_{T S V}=15 \mathrm{fF}\right)$.

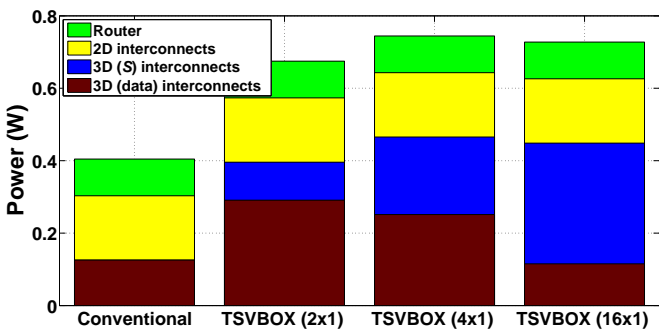

(b) Uniform $\left(C_{T S V}=500 \mathrm{fF}\right)$.

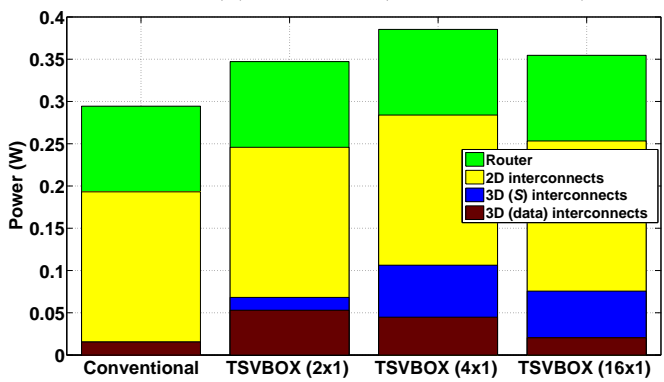

(e) Uniform $\left(C_{T S V}=15 \mathrm{fF}\right)$.

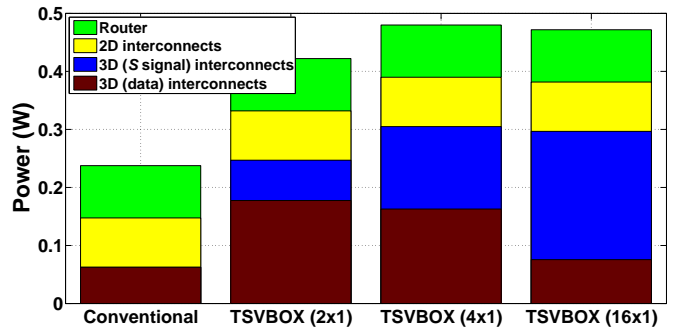

(c) Hotspot $\left(C_{T S V}=500 \mathrm{fF}\right)$.

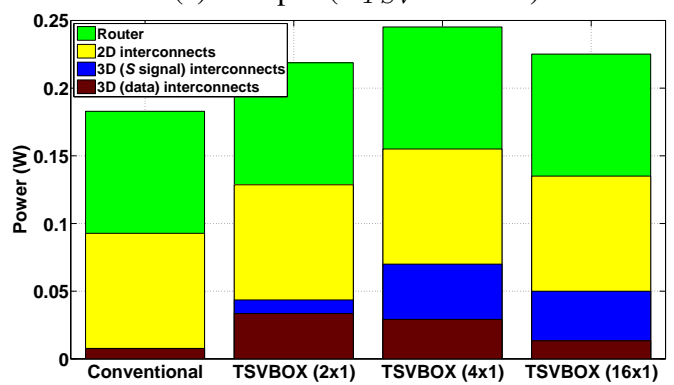

(f) Hotspot $\left(C_{T S V}=15 \mathrm{fF}\right)$.

Fig. 25: Power comparison for different TSV technologies, i.e. different TSV capacitances.

the number of transistors in its circuit. Since the driver inverter has two transistors, so we assume also it has half of the area of the MUX but we should account for the sizing of the $S$ signal driver (the sizes of the PMOS and NMOS transistors), therefore $A_{d r-S} \approx \frac{A_{M U X}}{4} \cdot\left(3 K_{N-S}\right)$, where $K_{P-S}=2 K_{N-S}$ to get equal charging and discharging currents during driver operation for $65 \mathrm{~nm}$ technology. The fab constants; $\alpha, D_{o}$, and $f_{T S V}$ are assumed to have the same values mentioned in [1], so $\alpha=2, D_{o}=0.004 \mathrm{~mm}^{2}$, and $f_{T S V}=10 \mathrm{ppm}$. Finally, $\mathrm{M}$ is assumed as a parameter taking the values; $4 \times 4,8 \times 8$, and $16 \times 16$.

Since die area is very large, the effect of TSV multiplexing area overhead is not high, and the normalized die yield in Fig. 26 is very close to 1 , showing no improvement in die yield but very small degradation. This degradation is increasing with the number of layers used, and it is only clear for large $\mathrm{M}^{6}$.

The enhancement in TSV yield is dependent mainly on the number of TSVs. In Fig. 27, the TSV yield is always enhanced as long as the number of TSVs is reduced, which was previously proved mathematically in Subsection VIII-A. The enhancement in TSV yield is boosted for large number of 3D stack layers. Extra boosting, comes from the data bus width, which reflects the total number in TSV yield equation (Eq. 21). As fewer as the total number of TSVs used (smaller $N_{B W}$ ), the greater will be the TSV yield. The increase in TSV yield with reduction in TSV count used is a very strong exponential relation. As shown in Fig. 27c, when the mesh size is $16 \times 16$, and $N_{B W}=1024$, there are $16 \times 16 \times 2 \times 1024$ which is about half million TSVs between two layers, the enhancement in TSV yield will be enormous; $\approx 86.746$ million

\footnotetext{
${ }^{6}$ Note that the same simulations were done for $D_{T S V}=40$ and the normalized die yield were larger than 1 for all cases we tried, but we show only the case of $D_{T S V}=1$ as it conforms with ITRS trends.
}

times than the TSV yield without multiplexing.

As depicted from Eq. 30 the normalized overall W2W yield is affected by all the parameters affecting both die and TSV yields. In Figs. 28a 28b 28c, although the die yield was less than 1, the overall yield is still enhanced; thanks to TSV yield.

Since the overall fabrication yield is improved for all the cases we tried, the fabrication cost will be reduced, as depicted from Eq. 31. Though, we do not show the results because they are only the reciprocal values of the overall W2W yield figures.

\section{CONCLUSIONS}

In this paper, the timing requirements analysis of the TSVBOX-based 3D NoC are investigated and its design methodology is presented. Using the proposed methodology, a $4 \times 4 \times 4$ mesh topology $3 \mathrm{D}$ NoC is modeled using SystemCA to verify various aspects of the target design. Analytical expressions for different 3D interconnect delays were derived using Elmore-delay estimations. The analytical delay models are verified using SystemC-A simulations and the error for different signals delays does not exceed $7.5 \%$, indicating rather acceptable accurate estimations of our proposed models.

Thereafter, performance comparisons in terms of average delay and throughput are conducted to investigate the direct effects of TSV multiplexing on these two metrics. We show that the TSVBOX does not affect 3D NoC performance under some conditions dependent on the application traffic pattern, clock frequency, and the required multiplexing ratio. The TSVBOX shows very close performance compared to the conventional case especially in case of Hotspot traffic pattern which models wide range of on chip processing applications. The side effects of TSVBOX on power consumption is studied also. We show that the TSVBOX power consumption becomes closer and closer to the conventional one as the TSV dimensions gets 


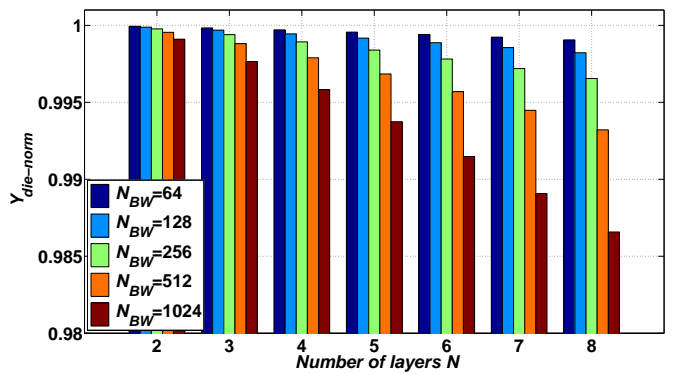

(a) $Y_{\text {die-norm }}(\mathrm{M}=4 \times 4)$.

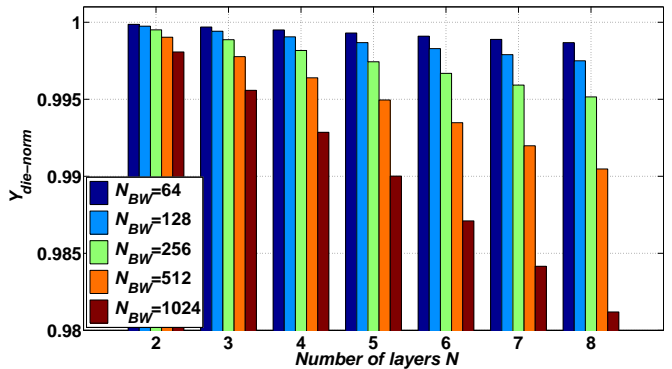

(b) $Y_{\text {die-norm }}(\mathrm{M}=8 \times 8)$.

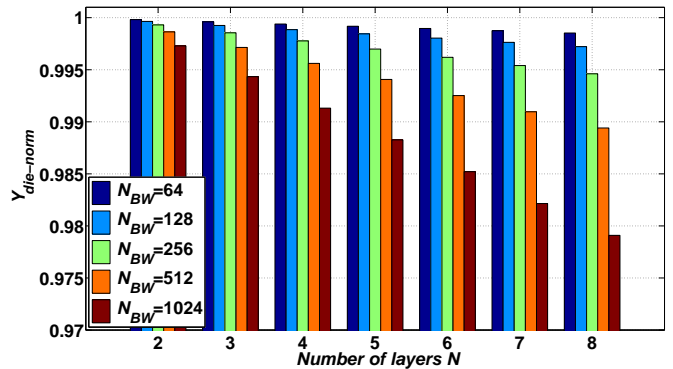

(c) $Y_{\text {die-norm }}(\mathrm{M}=16 \times 16)$.

Fig. 26: Normalized die yield under different data bus widths, number of 3D stack layers, and mesh sizes.

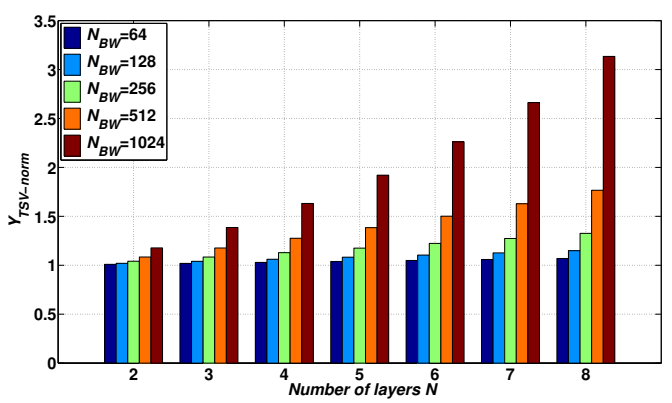

(a) $Y_{T S V-n o r m}(\mathrm{M}=4 \times 4)$.

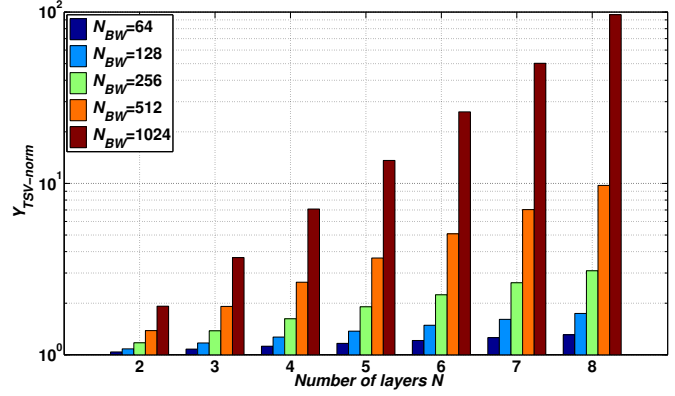

(b) $Y_{T S V-n o r m}(\mathrm{M}=8 \times 8)$.

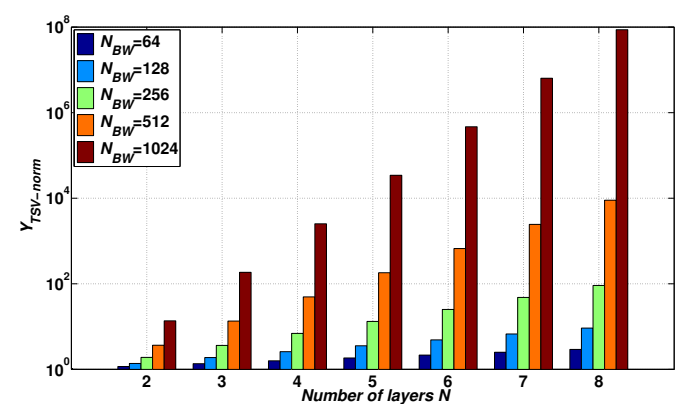

(c) $Y_{T S V-n o r m}(\mathrm{M}=16 \times 16)$.

Fig. 27: Normalized TSV yield under different data bus widths, number of 3D stack layers, and mesh sizes.

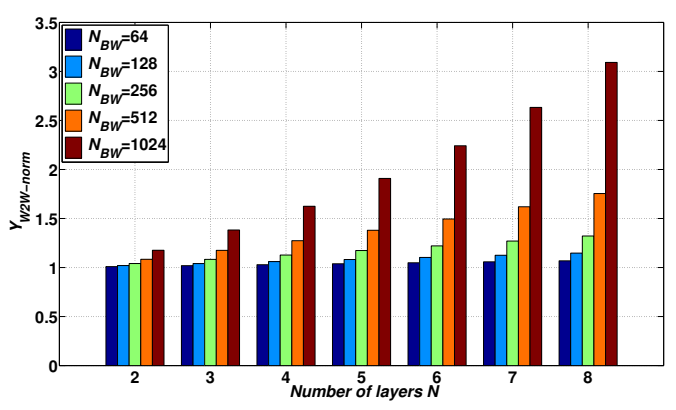

(a) $Y_{W 2 W-n o r m}(\mathrm{M}=4 \times 4)$.

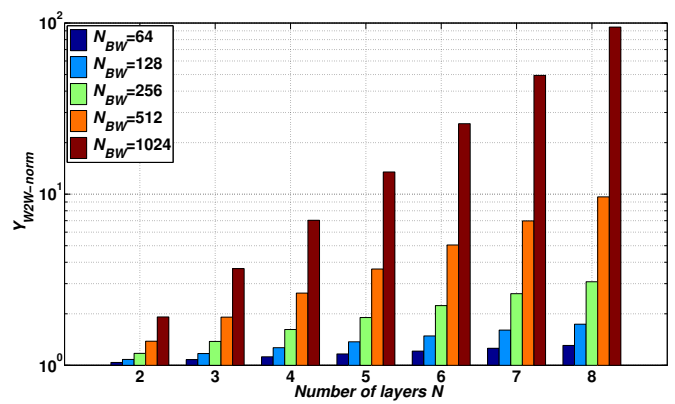

(b) $Y_{W 2 W-\text { norm }}(\mathrm{M}=8 \times 8)$.

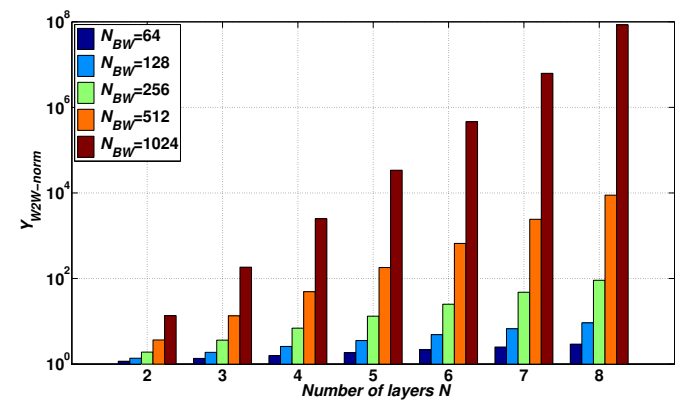

(c) $Y_{W 2 W-\text { norm }}(\mathrm{M}=16 \times 16)$.

Fig. 28: Normalized overall W2W yield under different data bus widths, number of 3D stack layers, and mesh sizes.

smaller which reflects the adaptability of the TSVBOX for the trend of TSV fabrication technology which intents to reduce TSV dimensions and hence its parasitic capacitance as well.

Also, we have investigated the effect of the design methodology on yield and cost. Though, the TSVBOX adds extra circuitry, which might need large sized drivers, the effect on area was minimal and still it can improve enormously the overall yield and reduce fabrication cost.

\section{APPENDIX A}

PROOFS OF EQS. 18, 19, AND 20

For simplicity, this analysis is done assuming an even number of the number of 3D stack layers ( $N$ is even).

\section{A. Performance analysis for Transpose traffic}

In Transpose traffic pattern, each node located at $(X, Y, Z)$ coordinates sends all its traffic to the node located at $\left(X_{S I Z E}-X-1, Y_{S I Z E}-Y-1, Z_{S I Z E}-Z-1\right)$ [36]. Therefore, nodes located in layer $L_{0}$ and layer $\left.L_{N-1}\right]^{7}$ send packets to each other, so do layer $L_{1}$ and layer $L_{N-2}$, etc.. According to that, packets from each pair of layers encounter the same extra waiting cycles in TSVBOX-based 3D NoC. The extra delay cycles for each pair can be calculated as follows: For $\left(L_{0}, L_{N-1}\right)$ pair, packets make $(N-1)$ hops going up from $L_{0}$ to $L_{N-1}$ or down from $L_{N-1}$ to $L_{0}$. According to Eq. 15 .

${ }^{7}$ We can consider $L_{0}$ as the first layer (layer \#0; usually it is the bottommost layer) and $L_{N-1}$ as the last layer (layer $\# N-1$; usually it is the top-most layer) in the 3D stack. 
those packets suffer from extra waiting cycles (EWCs):

$$
\left.E W C s\right|_{\left(L_{0}, L_{N-1}\right)}=N_{P K T} \cdot\left(n \cdot N_{M U X}-1\right) \cdot(N-1)
$$

Repeating the same for $\left(L_{1}, L_{N-2}\right)$ pair, we can deduce that packets traverse between these two layers suffer EWCs:

$$
\left.E W C s\right|_{\left(L_{1}, L_{N-2}\right)}=N_{P K T} \cdot\left(n \cdot N_{M U X}-1\right) \cdot(N-3)
$$

In general for any pair $\left(L_{m}, L_{N-m-1}\right)$, the EWCs:

$$
\left.E W C s\right|_{\left(L_{m}, L_{N-m-1}\right)}=N_{P K T} \cdot\left(n \cdot N_{M U X}-1\right) \cdot(N-2 m-1)
$$

The overall average EWCs $\left(E W C_{a v g}\right)$ is the sum of the EWCs of all pairs multiplied by 2 (because each layer of any pair has the same EWCs) and divided by $N-1(N-1$ represents the maximum vertical hops) for the sake of averaging

$$
\begin{array}{r}
E W C_{\text {avg-Transpose }}=2 \cdot N_{P K T} \cdot\left(n \cdot N_{M U X}-1\right) . \\
{\left[\left(\frac{N}{2}\right) \cdot N-(1+3+5+\ldots+(N-1)]\right.} \\
E W C_{\text {avg-Transpose }}=2 \cdot N_{P K T} \cdot\left(n \cdot N_{M U X}-1\right) . \\
{\left[\left(\frac{N^{2}}{2}\right)-\frac{N}{4} \cdot(N)\right]} \\
E W C_{\text {avg-Transpose }}=2 \cdot N_{P K T} \cdot\left(n \cdot N_{M U X}-1\right) \cdot \frac{N^{2}}{4}
\end{array}
$$

\section{B. Performance analysis for Uniform traffic}

In Uniform traffic pattern, each node uniformly distributes its traffic to all other nodes in the network with equal probabilities. Therefore, each node sends $\frac{N_{P K T}}{N}$ on average to each layer including its own layer. Of course the fraction $\frac{N_{P K T}}{N}$ that is directed to the node's co-layer nodes does not suffer EWCs while others going to other layers do. Just like the previous case of Transpose traffic, the EWCs value depends on the layer location and it is the same per pair of layers, i.e. $E W C s_{L_{0}}=E W C s_{L_{N-1}}, E W C s_{L_{1}}=E W C s_{L_{N-2}}$, etc.. The EWCs of $\left(L_{0}, L_{N-1}\right)$ pair is

$$
\begin{array}{r}
\left.E W C s\right|_{\left(L_{0}, L_{N-1}\right)}=\frac{N_{P K T}}{N} \cdot\left(n \cdot N_{M U X}-1\right) . \\
(1+2+3+\ldots+(N-1)) \\
=\frac{N_{P K T}}{N} \cdot\left(n \cdot N_{M U X}-1\right) \cdot\left(\frac{N(N-1)}{2}\right)
\end{array}
$$

Doing the same for $\left(L_{1}, L_{N-2}\right)$ pair

$$
\begin{array}{r}
\left.E W C s\right|_{\left(L_{1}, L_{N-2}\right)}=\frac{N_{P K T}}{N} \cdot\left(n \cdot N_{M U X}-1\right) . \\
(1+1+2+3+\ldots+(N-2)) \\
=\frac{N_{P K T}}{N} \cdot\left(n \cdot N_{M U X}-1\right) \cdot\left(1+\frac{(N-1)(N-2)}{2}\right)
\end{array}
$$

Continuing in the same way, EWCs for $\left(L_{\frac{N}{2}-1}, L_{\frac{N}{2}}\right)$ pair

$$
\begin{array}{r}
\left.E W C s\right|_{\left(L_{\frac{N}{2}-1}, L_{\frac{N}{2}}\right)}=\frac{N_{P K T}}{N} \cdot\left(n \cdot N_{M U X}-1\right) . \\
{\left[1+2+3+\ldots+\left(\frac{N}{2}-1\right)+\frac{\left(N-\left(\frac{N}{2}-1\right)\right)\left(N-\frac{N}{2}\right)}{2}\right]}
\end{array}
$$

Now the overall average EWCs $\left(E W C s_{a v g}\right)$

$$
\begin{array}{r}
E W C s_{\text {avg-Uniform }}=2 \cdot \frac{N_{P K T}}{N} \cdot\left(n \cdot N_{M U X}-1\right) . \\
{\left[\left(0+\frac{N(N-1)}{2}\right)+\left(0+1+\frac{(N-1)(N-2)}{2}\right)+\right.} \\
\left(0+1+2+\frac{(N-2)(N-3)}{2}\right)+\ldots+ \\
\left(\left(0+1+2+3+\ldots+\left(\frac{N}{2}-1\right)\right)+\right. \\
\left.\left.\left(\frac{\left(N-\left(\frac{N}{2}-1\right)\right)\left(N-\frac{N}{2}\right)}{2}\right)\right)\right]
\end{array}
$$

$$
\begin{array}{r}
E W C s_{\text {avg-Uniform }}=2 \cdot N_{P K T} \cdot\left(n \cdot N_{M U X}-1\right) . \\
{\left[\frac{1}{N} \cdot\left(\sum_{i=0}^{\frac{N}{2}-1} \frac{(N-i) \cdot(N-i-1)}{2}+\sum_{i=0}^{\frac{N}{2}-1} i \cdot\left(\frac{N}{2}-i\right)\right)\right]}
\end{array}
$$

\section{Performance analysis for Hotspot traffic}

In Hotspot traffic pattern, we assume that each node sends some part of its traffic $\left(h . N_{P K T}, 0<h<1\right)$ to some hotspot node/s and the rest of the traffic $\left((1-h) \cdot N_{P K T}\right)$ is uniformly distributed among all other nodes in the network. In our evaluation setup, it is assumed that there is a hotspot node in each layer where it is considered as a hotspot only for its co-layer nodes. According to that assumption, the analysis for Hotspot is the same as for Uniform, but $N_{P K T}$ is substituted by $\left((1-h) \cdot N_{P K T}\right)$. Thus, $E W C s_{\text {avg }}$ for Hotspot traffic can be calculated by the following equation:

$$
\begin{array}{r}
E W C s_{\text {avg-Hotspot }}=2 \cdot N_{P K T} \cdot\left(n \cdot N_{M U X}-1\right) . \\
{\left[\frac{(1-h)}{N} \cdot\left(\sum_{i=0}^{\frac{N}{2}-1} \frac{(N-i) \cdot(N-i-1)}{2}+\sum_{i=0}^{\frac{N}{2}-1} i \cdot\left(\frac{N}{2}-i\right)\right)\right]}
\end{array}
$$

\section{REFERENCES}

[1] M. Said, F. Mehdipour, and M. El-Sayed, Improving Performance and Fabrication Metrics of Three-Dimensional ICs by Multiplexing Through-Silicon Vias, 16th Euromicro Conference on Digital System Design (DSD), pp. 581-586, 2013.

[2] A. Papanikolaou, D. Soudris, and R. Radojcic, Three Dimensional System Integration, Springer, New York, 2011.

[3] I. Loi, S. Mitra, T. Lee, S. Fujita, L. Benini, A low-overhead Fault Tolerance Scheme for TSV-based 3D Network on Chip Links, IEEE/ACM International Conference on Computer-Aided Design (ICCAD), pp. 598-602, 2008.

[4] S. Pasricha, Exploring Serial Vertical Interconnects for 3D ICs, 46th ACM/IEEE Design Automation Conference (DAC), pp. 581-586, 2009.

[5] F. Miller, T. Wild, and A. Herkersdorf, TSV-Virtualization for Multi-Protocol-Interconnect in 3D-ICs, 15th Euromicro Conference on Digital System Design (DSD), pp. 374-381, 2012.

[6] M. Said, F. Mehdipour, and M. El-Sayed, Thermal Analysis of Three-Dimensional ICs, Investigating The Effect of ThroughSilicon Vias and Fabrication Parameters, Electrical Design of Advanced Packaging and Systems Symposium (EDAPS), pp. 165-168, 2013.

[7] M. Said, F. Mehdipour, N. Miyakawa, and M. El-Sayed, KeepOut-Zone Analysis for Three-Dimensional ICs, International 
Symposium on VLSI Design, Automation and Test (VLSI-DAT), pp. 1-4, 2014.

[8] M. Said, F. Mehdipour, k. Murakami, and M. El-Sayed, A Design Methodology for Performance Maintenance of $3 D$ Network-onChip with Multiplexed Through-Silicon Vias, 3rd ACM International Workshop on Manycore Embedded Systems (MES'15), June 2015 (to appear).

[9] S. Vangal et al., An 80-Tile 1.28TFLOPS Network-on-Chip in 65 nm CMOS, Proc. IEEE Intl Solid-State Circuits Conf. (ISSCC), pp. 98-99, 2007.

[10] BookSim 2.0 Users Guide: https://nocs.stanford.edu/cgibin/trac.cgi/wiki/Resources/BookSim.

[11] N. Jiang, D.U. Becker, G. Michelogiannakis, J. Balfour, B. Towles, ; D.E. Shaw, J. Kim, W.J. Dally, A detailed and flexible cycle-accurate Network-on-Chip simulator, IEEE International Symposium on Performance Analysis of Systems and Software (ISPASS), pp. 86-96, 2013.

[12] D.U. Becker and W.J. Dally, Allocator implementations for network-on-chip routers, Proceedings of the Conference on High Performance Computing Networking, Storage and Analysis, pp. $1-12,2009$.

[13] J. Duato, S. Yalamanchili, and L. Ni, Interconnection Networks, An Engineering Approach, Morgan Kaufmann, San Francisco, 2012.

[14] W.J. Dally and B. Towles, Principles and Practices of Interconnection Networks, Morgan Kaufmann Publishers, 2004.

[15] Y. Chen, D. Niu, and Y. Xie, Cost-effective integration of three dimensional(3D) ICs emphasizing testing cost analysis, IEEE/ACM International Conference on Computer-Aided Design (ICCAD), pp. 471-476, 2010.

[16] B.S. Feero, P.P. Pande, Networks-on-Chip in a ThreeDimensional Environment: A Performance Evaluation, IEEE Transactions on Computers, vol. 58, pp. 32-45, 2008.

[17] R. Jagtap, A Methodology for Early Exploration of TSV Interconnects in 3D Stacked ICs, Master thesis, TU Delft, 2011.

[18] G. Katti, M. Stucchi, K. De Meyer, and W. Dehaene, Electrical modeling and characterization of through silicon via for threedimensional ics, IEEE Transactions on Electron Devices, vol. 57, pp. 256-262, 2010.

[19] I. Savidis and E. Friedman, Closed-form expressions of 3-d via resistance, inductance, and capacitance, IEEE Transactions on Electron Devices, vol. 56, pp. 1873-1881, 2009.

[20] R. Weerasekera, M. Grange, D. Pamunuwa, H. Tenhunen, and L.-R. Zheng, Compact modelling of through-silicon vias (tsvs) in three-dimensional (3-d) integrated circuits, in IEEE International Conference on 3D System Integration (3DIC), pp. 1-8, 2009.

[21] C. A. Zeferino and A. A. Susin, SoCIN: A parametric and scalable network-on-chip, Proc. 16th Symposium on Integrated Circuits and Systems Design (SBCCI), pp. 169-175, 2003.

[22] http://www.systemc-ams.org/

[23] J. Rabaey, A. Chandrakasan, B. Nikolic, Digital Integrated Circuits, A Design Perspective, 2nd ed., Prentice Hall, New Jersey, 2003.

[24] N. Weste, D. Harris, CMOS VLSI Design, A Circuits and Systems Perspective, 4th ed., Addison Wesley, 2011.

[25] http://www.itrs.net/reports.html

[26] K. Banerjee, A. Mehrotra, Power dissipation issues in interconnect performance optimization for sub-180 nm designs, IEEE Symposium on VLSI Circuits, Digest of Technical Papers, pp. 12-15, 2002.

[27] J. Uyemura, CMOS Logic Circuit Design, Kluwer Academic Publishers, 1999

[28] M.P. Kennedy, M.A. Awan, and M.S. Asghar, A high frequency "divide-by-odd number" CMOS LC injection-locked frequency divider, Journal of Analog Integrated Circuits and Signal Processing, vol. 77 , pp. 415-421, 2013.

[29] K. Chandrasekar, Performance Validation of Networks on Chip,
Master thesis, TU Delft, 2009.

[30] P.P. Pande, C. Grecu, M. Jones, A. Ivanov, and R. Saleh, Performance Evaluation and Design Trade-Offs for Network on Chip Interconnect Architectures, IEEE Transactions on Computers, vol. 54, no. 8, pp. 1025-1040, 2005.

[31] D.R. Avresky, V. Shubranov, R. Horst, and P. Mehra, Performance Evaluation of the ServerNetR SAN under Self-Similar Traffic, 13th International and 10th Symposium on Parallel and Distributed Processing, pp. 143-147, 1999.

[32] D. E. Knuth, The Art of Computer Programming, 2nd ed., Addison-Wesley, 1981.

[33] H. Wang, X. Zhu, L.-S. Peh and S. Malik, Orion: A PowerPerformance Simulator for Interconnection Networks, Proc. MICRO, 2002, pp. 294-395.

[34] P.T. Wolkotte, G.J.M. Smit, N. Kavaldjiev, J.E. Becker, J. Becker, Energy Model of Networks-on-Chip and a Bus, Proc. of IEEE International Symposium on System-on-Chip, pp. 82-85, 2005.

[35] N. Jafarzadeh, M. Palesi, A. Khademzadeh, and A. AfzaliKusha, Data Encoding Techniques for Reducing Energy Consumption in Network-on-Chip, IEEE Transactions on Very Large Scale Integration (VLSI) Systems, vol. 22, pp. 675-685, 2014.

[36] S. Koohi, M. Mirza-Aghatabar, S. Hessabi, Evaluation of Traffic Pattern Effect on Power Consumption in Mesh and Torus Network-on-Chips, IEEE International Symposium on Integrated Circuits (ISIC), pp. 512-515, 2007.

[37] Gharan, Masoud Oveis, Power and chip-area aware networkon-chip simulation, Theses and dissertations, 2011.

[38] N. Concer, L. Bononi, M. Soulie, R.Locatelli, and L. P. Carloni, The Connection-Then-Credit Flow Control Protocol for Heterogeneous Multicore Systems-on-Chip, IEEE Transactions on Computer-Aided Design Of Integrated Circuits and Systems, vol. 29, JUNE 2010.

[39] A. Pullini, F. Angiolini, P. Meloni, D. Atienza, S. Murali, L. Raffo, G. De Micheli, and L. Benini, NoC Design and Implementation in $65 \mathrm{~nm}$ Technology. IEEE International Symposium on Networks-on-Chip (NOCS), 2007.

[40] P. K. Sahu, K. Manna, N. Shah, S. Chattopadhyay, Extending Kernighan-Lin Partitioning Heuristic for Application Mapping onto Network-on-Chip, Elsevier Journal of systems architecture, 2014.

[41] P. K. Sahu, S. Chattopadhyay, A Survey on Application Mapping Strategies for Network-on-Chip Design, Journal of Systems Architecture, vol. 59, pp. 60-76, 2013.

[42] N. Nethercote, P.J. Stuckey, R. Becket, S. Brand, G.J. Duck, G. Tack, MiniZinc: Towards a standard CP modelling language, Principles and Practice of Constraint Programming CP 2007, Lecture Notes in Computer Science, vol. 4741, pp. 529543, Springer (2007).

[43] http://www.minizinc.org/ 